\title{
Nonlinear Aeroelastic Framework Based on Vortex-Lattice Method and Corotational Shell Finite Element
}

\author{
Carlos Eduardo de Souza * and Roberto Gil Annes da Silva ${ }^{\dagger}$ \\ Technological Institute of Aeronautics, São José dos Campos, SP,Brazil \\ Carlos E.S. Cesnik * \\ University of Michigan, Ann Arbor, Michigan 48109-2140
}

\begin{abstract}
This paper presents a study on aeroelastic analyses of composite laminated wings subject to large displacements through the coupling of a nonlinear corotational shell finite element (FE) with an unsteady vortex-lattice method (UVLM) formulation. A FE implemented for the analysis of flat plates has been extended to model laminated composites with different lamina orientations. An UVLM formulation that is capable of coupling with this large displacement structural model is implemented. An explicit partitioned method is evaluated for the coupling of both models, using spline functions to interpolate information from the structural operator to the aerodynamic one, inside a Generalized- $\alpha$ time-marching solution. The resulting aeroelastic formulation provides a framework for the nonlinear aeroelastic analyses of structures made of composite material allowing the characterization of their nonlinear behavior and simulation of the limit-cycle oscillation response. Flat plate laminated wings designed for high flexibility and low flutter speed onset are used as investigation models. Effects of nonlinearities are easily observed in the numerical results, which are promising for expansion of the work and application to the analysis of more refined and complex composite flexible wings.
\end{abstract}

\section{Introduction}

A demand exist for analysis methodologies that take into account the nonlinear aeroelastic response of thin wings subject to large displacements in a generic unsteady flight condition with a good computational cost. The study of nonlinear problems in aeroelasticity, despite largely explored in the last decades, still offers opportunities for investigation. For example, the problem of nonlinear aeroelasticity of highly flexible aircraft associated to long span wings permits the use of beam models for the structural part of the problem. However, the interest in micro air vehicles (MAVs) turned the attention to the problem of the aeroelasticity of plate models. Another aspect is the use of more efficient materials, often in the form of fiber-reinforce composite materials.

The use of composite materials allows an overall increase in the structural efficiency of an airframe, with a better weight-to-thrust relation and large flexibility with positive safety factors. However, large displacements may compromise the aeroelastic behavior due to modifications in the aerodynamic loading of lifting surfaces, leading to strong non-linear effects such as geometric nonlinearities and flow separation. Furthermore, small thickness wings presenti nteresting analysis problems, bringing the importance of using a shell finite element (FE) in the structural part to adequately model such type of wings.

Different aerodynamic formulations were used in the large displacements problem investigation, including Peter's finite state methods ${ }^{1}$ or Navier-Stokes ${ }^{2}$ or Euler ${ }^{3}$ formulations. The use of unsteady vortex-lattice methods (UVLM) has also been attempted, because of its implementation simplicity and moderate reduction in computational costs. Large displacements and rotations are characteristics of the flapping wing problem, and the use of UVLM has been shown to be important because of the influence of the wake in the computation of propulsion forces ${ }^{4}$ in flight dynamics analyses. Another work ${ }^{5}$ applied the UVLM to the study of the aerodynamics of flat wings, rigid or with prescribed dynamic twist (pitching), but still without a flexible structural model. Banerjee ${ }^{6}$ investigated the aeroelasticity of MAV with the use of a 3-D, free wake UVLM coupled with a membrane model with displacements calculated using Fourier series. Wings are simulated as membranes supported by a rigid frame in forward flight at a constant angle of attack. Stanford and Beran ${ }^{7}$ couple the UVLM with a corotational FE to the investigation of flapping wings frame. The framework showed good results for structural response and aerodynamic forces prediction. Experimental work led by Heathcote ${ }^{8,9}$ to investigate the chordwise and spanwise flexibility in the flapping dynamics are references for some of the cited numerical studies of plate models suffering large displacements.

Application of UVLM to very flexible aircraft (VFA) wings is presented in ${ }^{10,11}$. The structural model is commonly represented by beam models, like a elastic beam coupled with rigid-body rotations ${ }^{12}$ or geometrically-nonlinear com-

*Doctoral candidate (carloses@ita.br), Department of Aeronautical Engineering, Senior Member,AIAA.

$\dagger$ Professor (gil@ita.br), Department of Aeronautical Engineering

‡Professor (cesnik@umich.edu), Department of Aerospace Engineering, Fellow, AIAA. 
posite beam ${ }^{11}$. For the structural modeling, the capability to model composite shells is part of the desired design goals. The use of finite difference approach for shell modeling ${ }^{13}$ does not allow a direct modeling of local characteristics of a composite-made wing. The use of a corotational methodology $7,14,15$ allows the simulation of composite plates wings, where aspect ratio considerations used for formulation of beam elements are not valid.

In ${ }^{16}$ a flat plate model was tested in a subsonic wind-tunnel. The experimental setup aimed identifying aeroelastic characteristics of the model and, in particular, the flutter onset. It showed a good correlation between the experimentally identified and predicted flutter mode shape and onset speed. A large displacement LCO was observed in the post flutter behavior or under certain flow perturbations. Further aeroelastic investigation in the direction of large displacements modeling have been motivated by the observed LCO characteristics.

This paper extends the above efforts by addressing laminated composite material and the stability of flat plate wings in forward flight, including stall effects. The "University of Michigan's Nonlinear Membrane Shell Solver" (UM/NLAMS ${ }^{14}$ ) is used in the computational structural dynamics (CSD) analysis side of the aeroelastic problem. This solver has been extended to include laminated composite materials. A UVLM formulation has been implemented and augmented with a stall model. The resulting nonlinear aeroelastic framework has similar complexity and computational costs from both structural and aerodynamics modeling.

The goals of this work are:

I. Develop an efficient nonlinear aeroelastic framework dedicated to the investigation of orthotropic material influence in the nonlinear aeroelastic response of very flexible composite wings.

II. Investigate the use of a stall model in the prediction of the LCO behavior.

III. Investigate the forward flight reponse at different flight conditions and material orientation using the proposed formulation.

In the next sections, the aeroelastic formulation is described, including the structural and aerodynamic models. Considerations about the numerical implementation are given, before showing numerical studies.

\section{Aeroelastic formulation}

In the present work the aeroelastic formulation is built for the analysis of flat wings subjected to large displacements. The aerodynamic and structural models are assembled independently, and coupling schemes are necessary to exchange information between both models. With that in mind, the aeroelastic equation of motion is defined as

$$
\mathbf{M} \ddot{\mathbf{x}}+\mathbf{C} \dot{\mathbf{x}}+\mathbf{K x}=\mathbf{f}_{s},
$$

where $\mathbf{M}, \mathbf{C}$ and $\mathbf{K}$ are the structural mass, damping and stiffness matrices, $\mathbf{x}$ is the displacement vector including translational and rotational degrees of freedom. The external forces on the structural model are obtained from the aerodynamic loading distribution, interpolated to the structural grid points. The right-hand side is a function of the nodal displacements $\mathbf{x}_{a}$ and velocities $\dot{\mathbf{x}}_{a}$ of the aerodynamic model, the interpolation matrix $\mathbf{G}_{a s}$ and the vorticity strength distribution $\Gamma$ :

$$
\mathbf{f}_{s}=\mathbf{G}_{a s}\left(\mathbf{x}, \mathbf{x}_{a}\right) \mathbf{f}_{a}\left(\dot{\mathbf{x}}_{a}, \mathbf{v}_{i n f}, \Gamma, t\right) .
$$

The explicit dependence on the vorticity distribution comes from the vortex lattice formulation, in which the vorticity distribution on the wake is used to calculate the vorticity distribution on the lifting surface, and subsequently, the pressure distribution. The aerodynamic force on each aerodynamic panel is calculated at a control point placed at $3 / 4$ chord in the panel, being obtained from the resultant pressure value and area $S_{i}$ of each panel $i$ :

$$
\mathbf{f}_{a i}=p_{i}\left(\dot{\mathbf{x}}_{a}, \Gamma_{i}, t\right) S_{i} .
$$

The aerodynamic model and the aerodynamic forces calculation are detailed in section IV..

The generalized- $\alpha$ method is employed to integrate the nonlinear aeroelastic equation of motion. It is a secondorder accurate unconditionally stable algorithm, and intended for low and high-frequency dissipation ${ }^{17}$, under definition of a single parameter $\rho_{\alpha}$. It uses similar displacement and velocity updates as in the the Newmark method. The system's equation is then rewritten with the algorithm parameters $\alpha_{f}$ and $\alpha_{m}$ (obtained from $\rho_{\alpha}$ ) in the following way:

$$
\mathbf{M} \ddot{\mathbf{x}}_{n+1-\alpha_{m}}+\mathbf{C} \dot{\mathbf{x}}_{n+1-\alpha f}+\mathbf{R}_{n+1-\alpha_{f}}=\mathbf{f}_{s}\left(t_{n+1-\alpha_{f}}\right) .
$$

The internal forces term can be written as

$$
\mathbf{R}_{n+1-\alpha_{f}}=\left(1-\alpha_{f}\right)\left(\mathbf{R}_{n}+\mathbf{K}_{t} \delta \mathbf{x}\right)+\alpha_{f} \mathbf{R}_{n} .
$$

where the internal forces vector at step $n+1$ depends on the previous step vector and the tangent stiffness matrix: $\mathbf{R}_{n+1}=\mathbf{R}_{n}+\mathbf{K}_{t} \delta \mathbf{x}$. For an initial condition in which the structure is at rest, $\mathbf{R}_{n}$ is null, and at any other condition the vectors are updated with the previous time step values. The solution for the displacement in the next step, $\delta \mathbf{x}$ is obtained using the effective stiffness matrix $\mathbf{K}_{e f}$ and the effective load vector $\mathbf{R}_{h}$, through the linear equation

$$
\mathbf{K}_{e f} \delta \mathbf{x}=\mathbf{R}_{h}
$$


A corrector procedure is included inside the time step, where a residual force $\Delta \mathbf{R}_{h}$ is used instead of $\mathbf{R}_{h}$ in Eq. (6). The terms in the above expressions are detailed in reference ${ }^{14}$. The structural matrices used above are obtained from the FE formulation, described in section III..

\section{A. Reference frames}

Multi-body dynamics concepts are essential when dealing with large rotational and translational displacements in a moving frame. Transformations between the different coordinate systems are used in the definition of the corotational formulation and in the definition of velocities vectors in the aerodynamic formulation. Some basic transformations involving large rotations are presented here, following ${ }^{18}$ and ${ }^{19}$. The different reference frames and coordinate systems are defined in Fig. 1: (1) an inertial fixed frame of reference $I F$, defined by vectors $\left[\mathbf{e}_{1}^{I}, \mathbf{e}_{2}^{I}, \mathbf{e}_{3}^{I}\right] ;$ (2) a coordinate system fixed to the body at its initial position, $B F_{0}$, defined by vectors $\left[\mathbf{e}_{1}^{B 0}, \mathbf{e}_{2}^{B 0}, \mathbf{e}_{3}^{B 0}\right] ;(3)$ a coordinate system fixed following the body, $B F$, that rotates and translates in relation to the inertial system $\left[\mathbf{e}_{1}^{B}, \mathbf{e}_{2}^{B}, \mathbf{e}_{3}^{B}\right] ;$ and (4) various local point frames $P F,\left[\mathbf{e}_{1}^{P}, \mathbf{e}_{2}^{P}, \mathbf{e}_{3}^{P}\right]$, that can be defined in both structural or aerodynamic models: at a nodal point in the structural model or at the $3 / 4$ chord point on an aerodynamic panel, for example.

The moving reference frames are updated at each time and sub-iterations steps, based on prescribed body motions defined instantaneously for all the independent degrees of freedom: translations $X_{1}, X_{2}$ and $X_{3}$, and rotations $\phi_{1}, \phi_{2}$ and $\phi_{3}$. These rotations form the rotation vector $\Phi$, so that each component represent a rotation around each $I F$ unit vector. The velocity of the $B F$ origin is given by the sum of the linear body velocity and the tangential velocity obtained from the rotational angular velocity:

$$
\mathbf{v}_{B 0}=\dot{\mathbf{X}}+\boldsymbol{\omega} \times \mathbf{X}
$$

where $\boldsymbol{\omega}$ is the $B F$ angular velocity vector. It is composed by the time derivatives of the rotation vector components: $\boldsymbol{\omega}=\left\{\dot{\phi}_{1}, \dot{\phi}_{2}, \dot{\phi}_{3}\right\}^{T}$.

The vector normal to the deformed surface at an arbitrary point on the wing, expressed in the $B F$, is given by $\mathbf{n}_{P}^{B}=n_{P i} \mathbf{e}_{B i}$. To represent the same vector in the $I F$, the following transformation is used:

$$
\mathbf{n}_{P}^{I}=\mathbf{T}_{I B} \mathbf{n}_{P}^{B},
$$

where $\mathbf{T}_{I B}$ is the matrix that transforms the coordinates from the $B F$ to the $I F$. This transformation matrix can be expressed using the Rodriguez formula ${ }^{19}$ :

$$
\mathbf{T}_{I B}=\mathbf{I}+\tilde{\mathbf{S}} \sin \varphi+2 \tilde{\mathbf{S}}^{2} \sin ^{2} \frac{\varphi}{2} .
$$

where $\mathbf{I}$ is the $3 \times 3$ identity matrix, $\tilde{\mathbf{S}}$ is the skew-symmetric (or antisymmetric) matrix of the rotation vector $\Phi=$ $\left\{\phi_{1}, \phi_{2}, \phi_{3}\right\}^{T}$, and $\varphi$ is the magnitude of rotation so that $\Phi=\varphi \bar{\Phi}$.

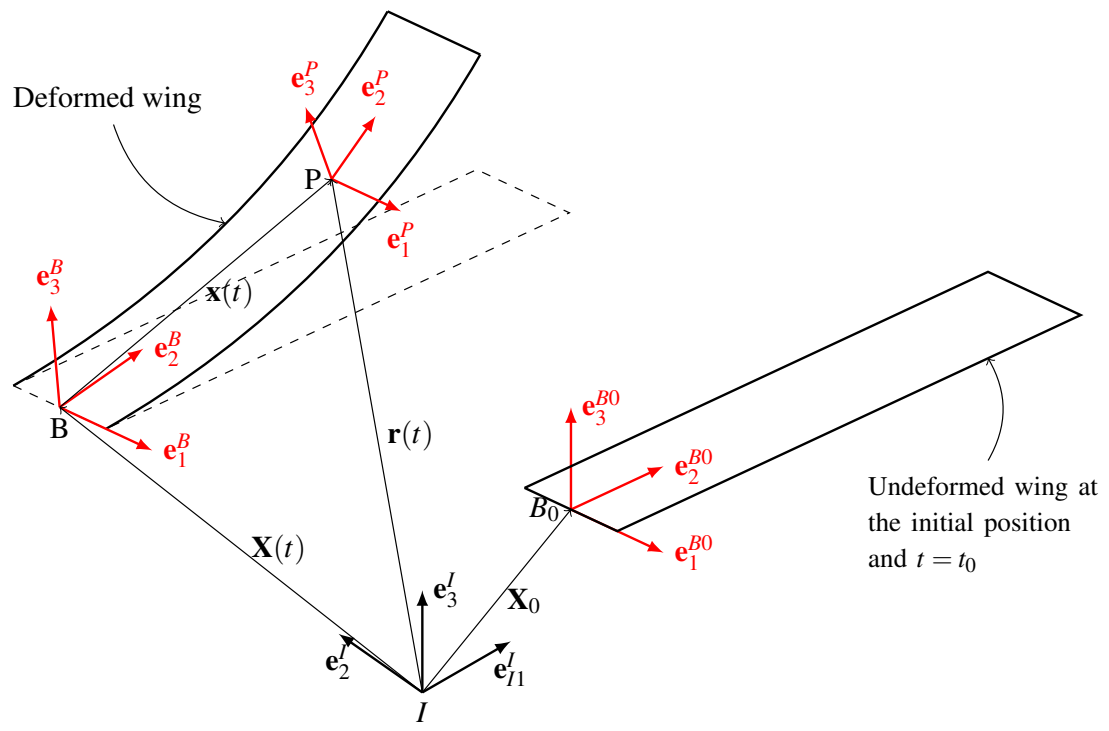

Figure 1. Basic coordinate systems for the present work.

\section{B. Interpolation schemes}

The interpolation method used is the thin-plate splines (TPS) method, which assume coincident surfaces supporting both structural and aerodynamic grids ${ }^{20}$. The TPS method shows to be robust and accurate ${ }^{21}$, specially for the large displacements involved in the simulation. 
The representation of a scalar quantity that is initially given in one set of points to other set whose points are in the same physical surface and same coordinate system is obtained through a transformation matrix $\mathbf{G}_{s}$. The conversion of displacements obtained in the structural model to the aerodynamic is depicted in Fig. 2(a). The points used to assemble the matrix $\mathbf{G}$ are the corner nodes from each structural finite element and the corner and central points of each aerodynamic panel, as seen in Fig. 2(a) and (b). The transformation is applied to each displacement component :

$$
\mathbf{a}_{a i}=\mathbf{G}_{s} \mathbf{a}_{s_{i}} .
$$

where the vector $\mathbf{a}_{s i}$ contains the components in the direction $i$ of displacement $\mathbf{q}_{s}$ at all the original points in the structural model and $\mathbf{a}_{a i}$ contains the component $i$ of the displacement $\mathbf{q}_{a}$ at all the aerodynamic interpolation points. The subscript $(\cdot)_{a}$ denotes that the quantity is related to the aerodynamic model and $(\cdot)_{s}$ to the structural one.

The same idea is used when returning the information of force distribution from the aerodynamic to the structural model. The difference now is that the information is passed from the $3 / 4$ chord point of each aerodynamic panel to the corner nodes of each structural finite element. Since the set of aerodynamic points used to assemble the matrix $\mathbf{G}_{a}$ is different from the previous case, a new matrix has to be created.

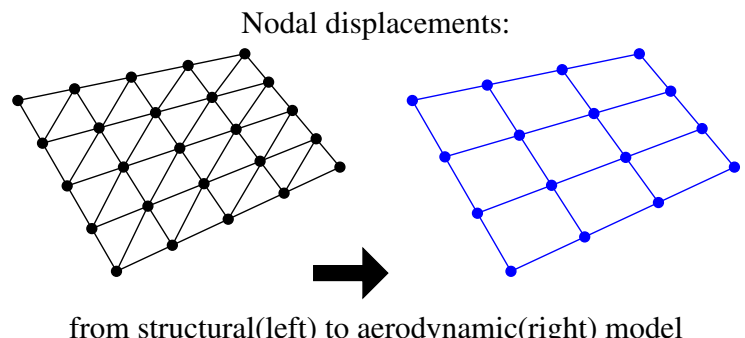

from structural(left) to aerodynamic(right) model

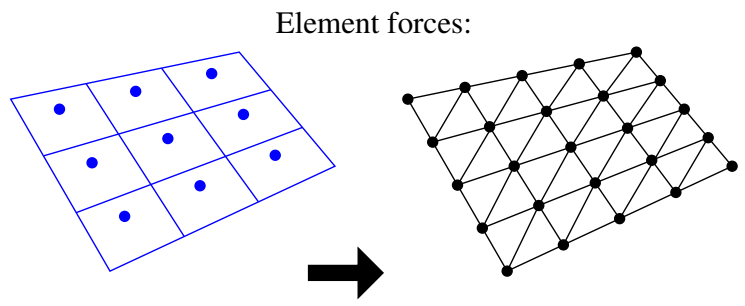

from aerodynamic(left) to structural(right) model

(a)

(b)

Figure 2. Schematic of the two interpolation steps performed in the aeroelastic frame showing the base points used to assemble the transformation matrices.

\section{Structural Model}

The nonlinear CSD is solved using the UM/NLAMS ${ }^{14,15}$. It has an expanded formulation for a co-rotational shell finite element (FE) presented in ${ }^{22}$ to the analysis of flapping wings. This FE has special features for the time response simulation and flight dynamics of the complete airframe, and its formulation is based on linear triangular shell elements. The linear matrices are assembled using plate theory, with decomposition of membrane and bending components and the coupling resultant, $\mathbf{K}_{b}, \mathbf{K}_{m}$ and $\mathbf{K}_{m b}$ :

$$
\mathbf{K}_{b}=\int \mathbf{B}_{b}^{T} \mathbf{D} \mathbf{B}_{b} d A_{e}, \quad \mathbf{K}_{m}=\int \mathbf{B}_{m}^{T} \mathbf{A} \mathbf{B}_{m} d A_{e}, \quad \mathbf{K}_{m b}=\int \mathbf{B}_{m}^{T} \mathbf{B} \mathbf{B}_{b} d A_{e}
$$

The resultant linear stiffness matrix is:

$$
\mathbf{K}_{e l}^{\text {shell }}=\left[\begin{array}{cc}
\mathbf{K}_{m} & \mathbf{K}_{m b} \\
\mathbf{K}_{m b} & \mathbf{K}_{b}
\end{array}\right]
$$

The information about laminated composites are all defined in a pre-processing step, and do not change along the simulation. The matrices $\mathbf{A}, \mathbf{B}$ and $\mathbf{D}$ are calculated for each different element properties defined in the model, using the expressions:

$$
A_{i j}=\sum_{k=1}^{n l} \bar{Q}_{i j}^{(k)}\left(z_{k+1}-z_{k}\right), \quad B_{i j}=\frac{1}{2} \sum_{k=1}^{n l} \bar{Q}_{i j}^{(k)}\left(z_{k+1}^{2}-z_{k}^{2}\right), \quad D_{i j}=\frac{1}{3} \sum_{k=1}^{n l} \bar{Q}_{i j}^{(k)}\left(z_{k+1}^{3}-z_{k}^{3}\right)
$$

where $\bar{Q}_{i j}^{(k)}$ are the elements of the lamina constitutive matrix given in the plate coordinate system, what is obtained from the material coordinate system (according to the fabric pattern) through a plane rotation using the angle $\theta_{k}$. This is the Classical Laminated Theory - CLT, and its formulation is found in many references, among then ${ }^{23}$.

On top of this linear plate formulation, the corotational method is used to obtain the nonlinear displacement. The complete formulation is described by ${ }^{14,15}$, and will not be repeated here. An important characteristic of this methodology is the need for the coordinate transformations, presented in section A., in order to describe geometric nonlinearities and large displacements in time. The reference frames used in the structural formulation are shown in Fig. 3, where a typical triangular shell finite element is in its initial (undeformed) and the current (deformed) configurations. The figure is basically the same as that in Fig. 1, but now emphasizing the nodal coordinate systems. 
The final equations of motion are written with respect to the body frame, $B F$. In the definition of the element formulation, the transformation matrix from this global frame to the inertial frame is used, given by $\mathbf{T}_{I B}$ (defined in Eq. (9)). Also, it is used the transformation matrix $\mathbf{T}_{B E_{o}}$, that relates the element coordinate system in the initial position to the deformed one in the $B F$. The element nonlinear structural matrices are all calculated inside a timemarching process that uses a Generalized - $\alpha$ integration algorithm, along with predictor-corrector procedures in the nonlinear solver. They are implemented inside the UM/NLAMS code, which description is also given in ${ }^{14}$.

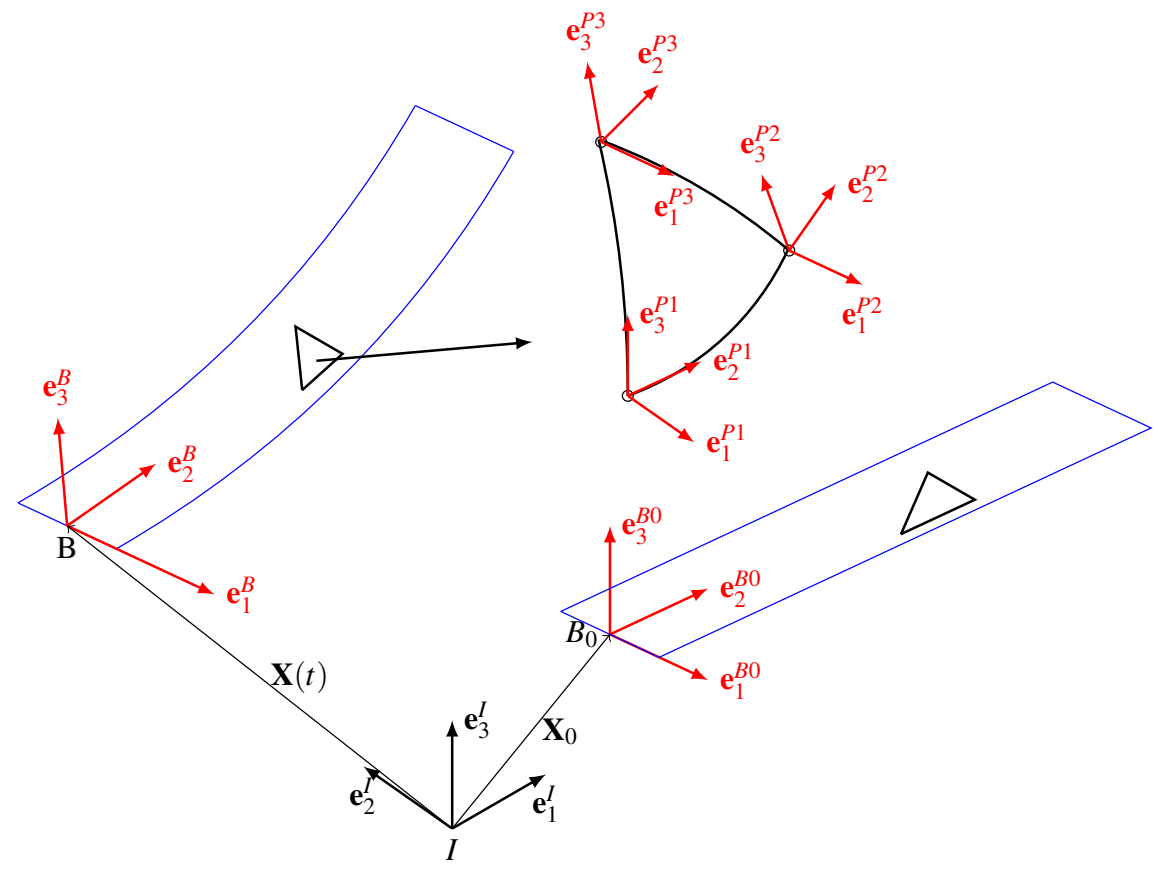

Figure 3. Coordinate systems for the structural model.

\section{Aerodynamic model}

The aerodynamic model applied to this investigation is an unsteady vortex lattice method (UVLM). This method is computationally simple and has been very well documented by a large number of authors. Reference ${ }^{24}$ presents a good description of the UVLM, including numerical procedures and the corresponding algorithms. Adaptation to the problem of large displacements in a 3D domain includes special procedures implemented in this work.

The vortex lattice method is based on the assumption of incompressible irrotational flow allowing the existence of a potential function that is a single scalar variable, namely the velocity potential. The gradient of such a quantity is the flow velocity, that is, $\mathbf{V}=\nabla \Phi$. Assuming incompressible flow and the velocity potential, velocity components and a time and space invariante density function, continuity equation is reduced to Laplace's equation as

$$
\nabla^{2} \Phi=0 .
$$

The solution of this linear partial differential equation requires the kwoledge of boundary contidions. Here it is assumed zero normal flow across the wing surface, represented by

$$
\nabla(\Phi+\mathbf{v}) \cdot \mathbf{n}=0
$$

where $\mathbf{v}$ is the surface's kinematic velocity and $\mathbf{n}$ is the vector normal to the lifting surface. The Laplace's equation is a full potential operator, not being obtained by any linearization assumption based on small disturbances. For this reason the nonlinear kinematic boundary condition relation presented before shall be considered, and it can be applied to the representation of lifting surfaces under large displacements. Since the Laplace equation is a linear operator, solution of this equation can be linearly combined in a superposition sense. Aerodynamic singularity functions (vortex, source, doublets) are solution of Laplace equation. The UVLM assumes the lifting surface can be represented by a vortex singularity distribution. The are two types of vortex singularity to be used in the UVLM: horseshoe vortex and vortex ring.

The vortex ring approach has an important characteristic that allows its use in unsteady formulations, that is, both the lifting surface and the wake are divided into vortex elements. Bound vortex elements are placed over the lifting surface while shed vortex elements are convected from the trailing edge forming the wake. Induced velocity flow field 
might convect the shed vortex elements allowing its roll up. Time marching solution for solving Laplace's equation assumes the lifting surface displacements as a function of time, or in other words, unsteady kinematic boundary conditions updating the position of all elements at each time step.

The lifting surface is divided into elements containing vortex ring singularities, as seen in Fig. 4. Vortex lines are superposed to a panel element forming the vortex ring. The forward line is placed at $25 \%$ element chord position, and the rearward line is placed at $25 \%$ chord of the next element. The lateral lines run on top of the element edges. This way, the collocation point is equally distant to both sides, but remains in the center of the vortex ring element.

The velocity induced by the closed curve $\mathrm{C}$ is expressed as an integral along the curve:

$$
\mathbf{w}_{c}=\frac{\Gamma}{4 \pi} \int_{C} \frac{d \mathbf{l} \times \mathbf{r}}{r^{3}} d C
$$

The induced velocity $\mathbf{w}_{i j}$ is the influence on a element $i$ by an element $j$, and is expressed as

$$
\mathbf{w}_{i j}==\left(\frac{1}{4 \pi} \int_{C j} \frac{d \mathbf{l} \times \mathbf{r}_{i}}{r_{i}^{3}}\right) \Gamma_{j}=\overline{\mathbf{w}}_{i j} \Gamma_{j}
$$

This integration can be substituted by a summation of the velocity induced by the four sides of the vortex ring by assuming an unit induced velocity due to the vortex ring element by means of Biot-Savart law, defined as,

$$
\overline{\mathbf{w}}_{i j}=\sum_{k=1}^{4}\left\{\frac{1}{4 \pi} \frac{\mathbf{r}_{p i} \times \mathbf{r}_{q i}}{\left|\mathbf{r}_{p i} \times \mathbf{r}_{q i}\right|^{2}}\left[\mathbf{r}_{0 i}\left(\frac{\mathbf{r}_{p i}}{r_{p i}}-\frac{\mathbf{r}_{q i}}{r_{q i}}\right)\right]\right\}_{k}
$$

where $p=2,3,4,1$ and $q=1,2,3,4$ in this order, representing the order of vortex ring corner nodes.

If the lifting surface is divided into $m$ vortex ring elements, the potential at a given control point placed at an element $i$ will the result of a linear sum of all the influences from other elements in the domain: bound and shed, the latter hereafter named as wake elements. Once the gradient in Eq. (15) is a linear operator, and can be split into two separate terms:

$$
\nabla \Phi=\nabla \Phi_{B}+\nabla \Phi_{w}
$$

where $\Phi_{B}$ is the potential of bound elements and $\Phi_{w}$ is the potential of the shed wake.

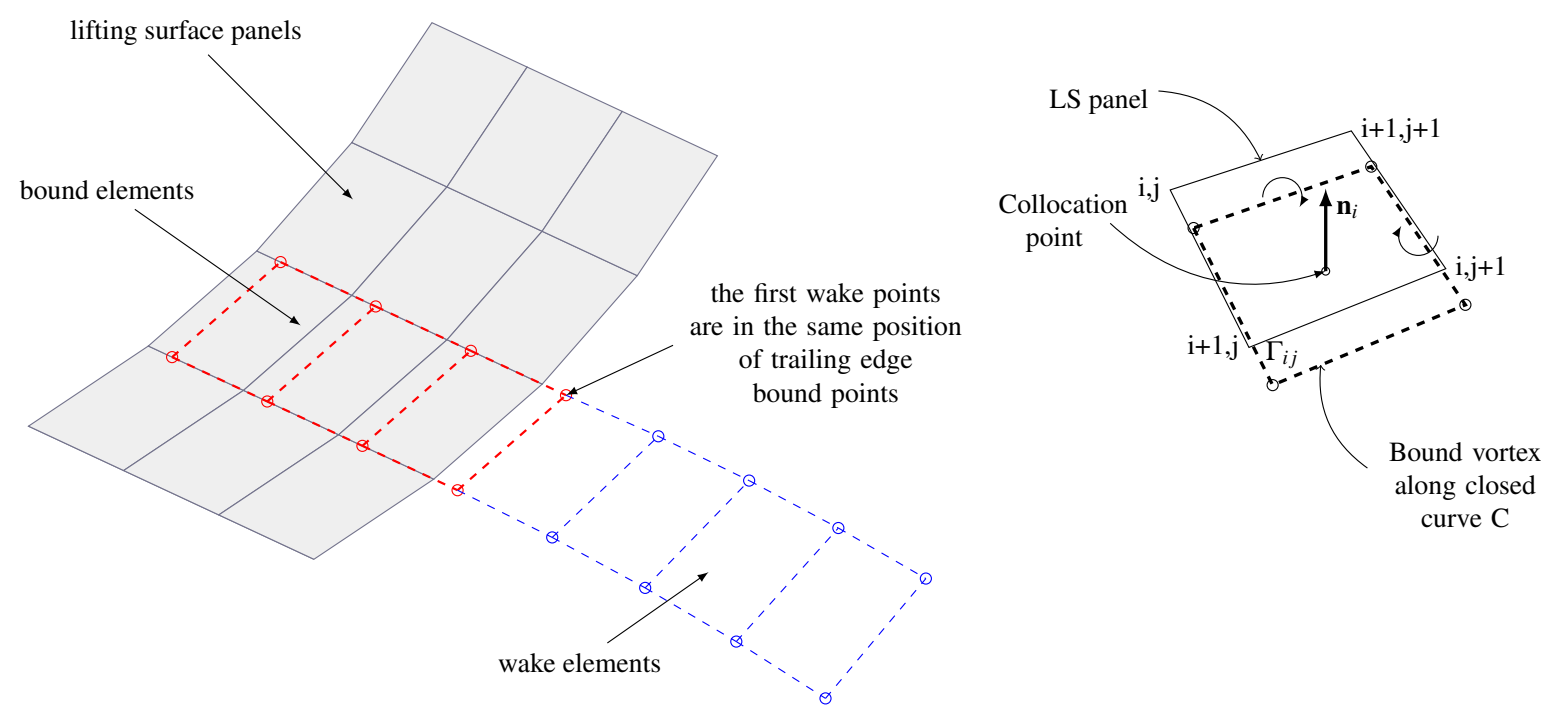

Figure 4. A vortex ring element imposed on a rectangular wing panel.

Defining the velocity induced by the wake as $\nabla \Phi_{w}=\mathbf{w}_{w}$, and $\mathbf{v}$ as an external flow velocity $-\mathbf{v}_{\text {ext }}$, Eq. (15) can now be rewritten as:

$$
\left(\nabla \Phi_{B}+\nabla \Phi_{w}+\mathbf{v}\right) \cdot \mathbf{n}=\left(\nabla \Phi_{B}+\mathbf{w}_{w}-\mathbf{v}_{\text {ext }}\right) \cdot \mathbf{n}=0
$$

The total local velocity is defined to include explicitly all velocities contribution at a given control point. The resulting velocity is composed by the wake induced velocity and the total induced velocity as

$$
\begin{gathered}
\mathbf{v}_{\text {res }}=\mathbf{w}_{w}-\mathbf{v}_{\text {tind }} . \\
6 \text { of } 21
\end{gathered}
$$


Taking into consideration that a panel is moving in an inertial system and that the lifting surface is also moving in relation to the body frame, the total induced velocity is actually computed as:

$$
\mathbf{v}_{\text {tind }}=\mathbf{v}_{\text {kin }}^{P}-\mathbf{v}_{\text {wind }}^{P},
$$

where $\mathbf{v}_{\text {wind }}$ is the velocity of a local wind (atmospheric) and $\mathbf{v}_{\text {kin }}$ is the kinematic velocity. The kinematic velocity is composed by the local velocity of the lifting surface due to deformation or imposed rotations, $\mathbf{v}_{\text {panel }}$, and body velocity due to an imposed movement $\mathbf{v}_{b o d y}$ :

$$
\mathbf{v}_{\text {kin }}^{P}=\mathbf{v}_{\text {panel }}^{P}+\mathbf{v}_{\text {body }}^{P} .
$$

Linear and angular body velocities are included in the above expression, as per the definition of $\mathbf{v}_{\text {body }}$ in Eq. (7). The superscript $P$ means that the above resulting velocity components have to be established in the panel reference frame. The calculation of the kinematic velocities depend on the moving frames formulation, explained in section A..

Finally, the expression for the resulting velocity is given by:

$$
\mathbf{v}_{\text {res }}=\mathbf{w}_{w}-\left(\mathbf{v}_{\text {panel }}^{P}+\mathbf{v}_{\text {body }}^{P}\right)+\mathbf{v}_{\text {wind }}^{P},
$$

where the negative sign at the kinematic velocity terms means that the equivalent flow velocity is in opposite direction of the panel movement, in the panel coordinate system.

With this resulting velocity defined, a linear equation system is assembled, where the bound element vorticity strength $\Gamma_{j}$ is the unknown:

$$
\left(\overline{\mathbf{w}}_{i j} \cdot \mathbf{n}_{j}\right) \Gamma_{j}=-\mathbf{v}_{r e s j} \cdot \mathbf{n}_{j} .
$$

In matrix notation, the above system is given by:

$$
\overline{\mathbf{A}} \mathbf{g}=\mathbf{f}_{v} .
$$

The matrix $\overline{\mathbf{A}}$ is the aerodynamic influence coefficients matrix, $\mathbf{g}$ is the vector of circulation strength at the control points of bound vortex elements, and $\mathbf{f}_{v}$ is the right-hand side vector. The time dependency is included in the boundary conditions by means of panel normal vector and wake position history, and relates to a geometry update at each time step.

\section{A. Free wake approach}

With the bound element vorticity computed from the solution of Eq. (25), the next step is the computation of free wake shed vorticity and vortex ring element spatial position. The physical explanation for the existence of a shed wake is given by Kelvin's Theorem, where it states that the total circulation in the flow field need to be zero. In other words, once a bound vorticity exists, a shed vorticity need also to exists in opposite direction such that the total vorticity present in the flow field remains constant and null.

The free wake solution also leads to "wake rollup" 24 , which relates do the fact that when the wake is set free to move according to the local induced velocity, it results in a rolling condition at the wake free edges. The induced velocity at a point " $i$ " due to the vorticity of a vortex-ring element $j$ can be defined using the element definition in Eq. (18), and is calculated after obtainng the vorticity strength on all lifting surfaces and wake elements from the solution of Eq. (26). It is a summation of velocities $\mathbf{w}_{B i j}$ (induced velocity on point $i$ by an bound element $j$ ), and velocities $\mathbf{w}_{\text {wik }}$ (induced velocity on point $i$ by a free element $k$ ):

$$
\mathbf{w}_{i}=\sum_{j} \mathbf{w}_{B i j}+\sum_{k} \mathbf{w}_{w i k}
$$

In the present implementation, where the body movements are expressed in an inertial frame, the induced displacement is added to the inertial displacement $\mathbf{u}_{I}$, so that the updated position of each wake point is then given by:

$$
\mathbf{x}_{w}=\mathbf{x}_{0}+\mathbf{u}=\mathbf{x}_{0}+\mathbf{u}_{I}+\mathbf{u}_{\text {ind }}
$$

where $\mathbf{x}_{0}$ is the initial point position, $\mathbf{u}_{I}$ is the inertial displacement, passed from the last bound element grid points, and $\mathbf{u}_{\text {ind }}=\mathbf{w} \Delta t$. This procedure, however, is time consuming, and the computational cost increases with longer wake sheets.

\section{B. Pressure and forces}

The pressure distribution is found using the unsteady Bernoulli equation. Following the notation of ${ }^{24}$, it is expressed as

$$
\frac{p_{r e f}-p}{\rho}=\frac{Q^{2}}{2}-\frac{v_{r e f}^{2}}{2}+\frac{\partial \Phi}{\partial t} \text { body }
$$


where $p$ is the local fluid pressure, $Q$ is the magnitude of the local fluid velocity, and the subscript $r e f$ indicates a reference field condition. This equation is based on the resulting velocity at each panel that depends on the lifting surface displacement history and on the solution of the potential equation, obtained from Eq. (26). The pressure difference between the upper and lower surfaces is then

$$
p_{r e f}-p=\rho\left[\left(\frac{v_{t}^{2}}{2}\right)_{u}-\left(\frac{v_{t}^{2}}{2}\right)_{l}+\left(\frac{\partial \Phi}{\partial t}\right)_{u}-\left(\frac{\partial \Phi}{\partial t}\right)_{l}\right]
$$

The terms of this equation are calculated as presented in $^{24}$. Considering the tangential derivative of thin airfoil potential, the tangential velocities $v_{t}$ in the $\mathbf{e}_{1}$ and $\mathbf{e}_{2}$ directions are defined from

$$
\begin{aligned}
& v_{t_{1}}=\mathbf{v}_{\text {res }} \cdot \tau_{1}\left( \pm \frac{\partial \Phi}{\partial \tau_{1}}\right)=\mathbf{v}_{\text {res }} \cdot \tau_{1}\left( \pm \frac{\Gamma_{i j}-\Gamma_{i-1, j}}{2 \Delta c}\right), \\
& v_{t_{2}}=\mathbf{v}_{r e s} \cdot \tau_{2}\left( \pm \frac{\partial \Phi}{\partial \tau_{2}}\right)=\mathbf{v}_{r e s} \cdot \tau_{2}\left( \pm \frac{\Gamma_{i j}-\Gamma_{i, j-1}}{2 \Delta b}\right),
\end{aligned}
$$

where the \pm sign stands for upper and lower surfaces, respectively, $\Delta c$ is the panel chord and $\Delta b$ is the panel span. From the relation between the potential jump and vorticity across the lifting surface and wake ${ }^{24}$, the velocity-potential time derivative is obtained as

$$
\pm \frac{\partial \Phi}{\partial t}= \pm \frac{\partial}{\partial t} \sum_{k=1}^{j} \frac{\Gamma_{k}}{2}
$$

A manipulation using definitions in Eqs. (31) and (32), yields a discretization of Eq. (30) as:3

$$
\Delta p_{k}=\rho\left\{\mathbf{v}_{r e s}(t) \cdot\left(\tau_{i} \Delta \Gamma_{i}\right)+\mathbf{v}_{r e s}(t) \cdot\left(\tau_{j} \Delta \Gamma_{j}\right)+\frac{\partial}{\partial t} \Gamma i, j\right\},
$$

where $\Delta \Gamma_{i}=\left(\Gamma_{i j}-\Gamma_{i-1, j}\right) / \Delta c_{i j}$ and $\Delta \Gamma_{j}=\left(\Gamma_{i j}-\Gamma_{i, j-1}\right) / \Delta b_{i j}$, and the $\Delta p_{k}$ is the pressure applied to the $k^{\text {th }}$ panel considering both tangential velocities potential derivatives. The resulting force vector at a panel is obtained simply by multiplying the pressure by the area and the panel normal vector:

$$
\mathbf{f}_{k}=\Delta p_{k} S_{k} \mathbf{n}_{k}
$$

The total force is obtained from a summation on all elements, and the force coefficients $c_{F}$ obtained by dividing this force by the total lifting surface are multiplyed by an equivalent dynamic pressure:

$$
\mathbf{c}_{F}=\frac{2}{\rho v_{r e f}^{2} S_{T}} \mathbf{f}_{T}
$$

where $S_{T}$ is the total area and $\mathbf{f}_{T}$ is the total force vector. This force coefficient vector is given in the same coordinate system used to define $\mathbf{n}_{k}$ in Eq. (34).

\section{Effective angle-of-attack}

The definition of an effective angle of attack is necessary for the later implementation of a stall model, since it relies on this information. Here, the local angle of attack is defined as the angle between the local resultant velocity and the panel orientation. This velocity vector is the same used at the core of the UVLM, defined in Eq. (21) and used in the RHS of the linear system of equations used to solve for the vorticity strength distribution. 


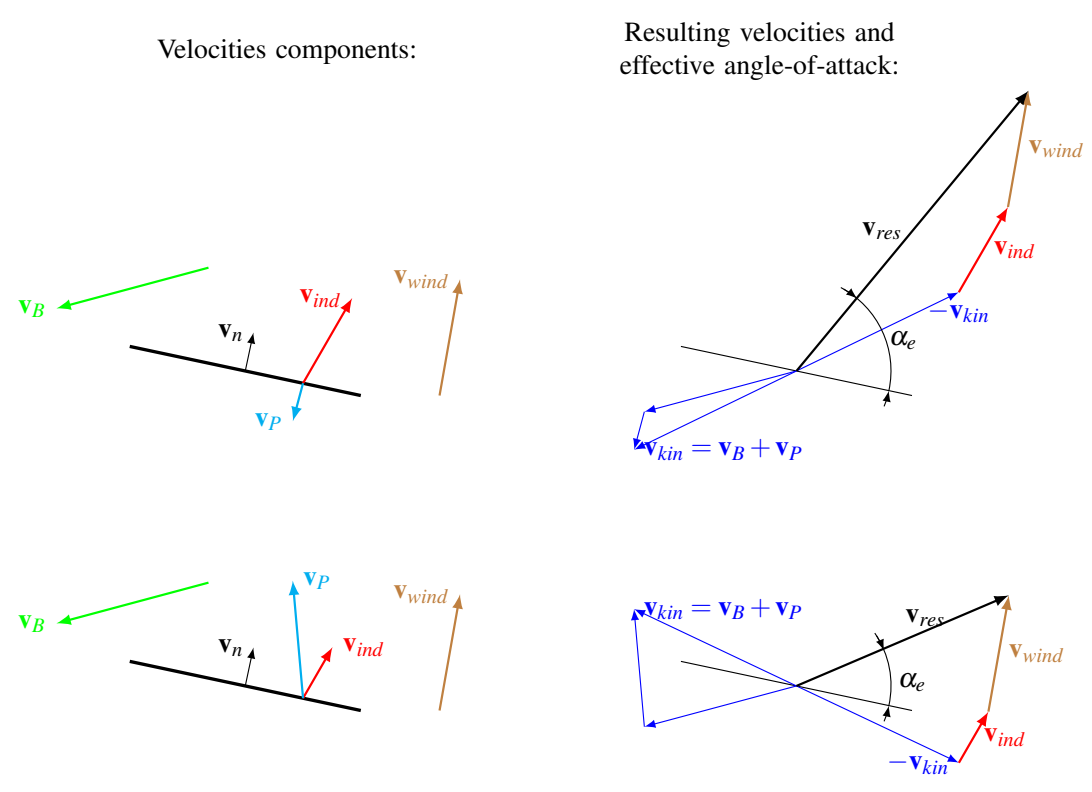

Figure 5. Definition of element resulting velocity and local effective angle of attack.

The effective angle of attack is then obtained from an internal product between the resulting velocity $\mathbf{v}_{\text {res }}$ and the panel chordwise direction:

$$
\alpha_{e}=\cos ^{-1}\left(\frac{\mathbf{v}_{\text {res }} \cdot \mathbf{e}_{1}}{\left|\mathbf{v}_{\text {res }}\right|\left|\mathbf{e}_{1}\right|}\right)
$$

\section{Stall model}

In forward flight of flexible wings, local angle of attack can achieve values above the profile static stall limit even for moderate displacements. This situation brings difficulties to the aeroelastic problem using UVLM due to the nature of stall. The stall is related with viscous flow separation over a lifting surface at high angles of attack. On the other hand, a potential based solution such as the UVLM is not an adequate model for representing separation effects.

As an approach applied to standard VLM, Ref. ${ }^{25}$ uses experimental data to take into account viscous effects that dominate the separated flow. A separated vortex ring element was implemented, covering an area that begins at the separation point and goes until the reattachment point. This methodology, however, requires preliminary studies about the wing profile being analyzed, since chordwise separation lines must be known. Also, in ${ }^{25}$ the simulations were all performed for steady stalled conditions, and is not applicable to dynamic simulation where dynamic stall behavior is dominant. Reference ${ }^{26}$ studies the histeretic behavior resultant from the use of different stall models. The main contribution to hysteretic behavior of the lift coefficient is not only the separeted flow effect. Theodorsen's potential flow model ${ }^{27}$ for a two dimensional oscillating thin plate indicated that the histeretic lift coefficient behavior is related to unsteady wake vorticity potential interference on bound vorticity.

Figure 6 shows the static $C_{L} \times \alpha$ curves for a flat plate wing with increasing aspect ratios compared to the $2 \pi$ reference line and a reference curve for a flat plate section. The results shown for the vortex lattice solution do not contain stall corrections. For higher aspect ratios and small angles of attack, the curves are very close to the $2 \pi$ line, what is not verified for $A R=4$. Increasing the angles, there is an increasing distance to the reference line, what is expected due to tip vortex effects. Comparing to the reference curve for thin plates, what is noticed is exactly that some stall effects are necessary to adjust the results.

For the sake of expediency regarding the implemented numerical aerodynamic model, the method of choice to overcome this limitation is based on an engineering approach. This approach consists in applying a relaxation factor to the $C_{P}$ obtained on a bound vortex-ring element after the VLM time-step solution. A proportional penalty factor $p_{s}$ is applied to the $C_{P}$ of an element $k$ :

$$
C_{P, \text { stall }}=p_{s} C_{P}, \quad \text { where } p_{s}=\left\{\begin{array}{l}
1, \text { if } \alpha_{e} \leq \alpha_{c u t} \\
\varepsilon_{\alpha} \frac{\alpha_{c u t}}{\alpha_{e}}, \text { if } \alpha_{e}>\alpha_{c u t}
\end{array}\right.
$$

and $\alpha_{e}$ is the bound element effective angle of attack defined in the previous section and $\alpha_{\text {cut }}$ is an arbitrary stall angle that has to be defined based on experiments or higher-order numerical methods. To complement the formula, a factor $\varepsilon_{\alpha}$, defined as the post-stall penalty factor, is used to adjust the response for larger angles. A schematic description of this process is shown in Fig. 7. 


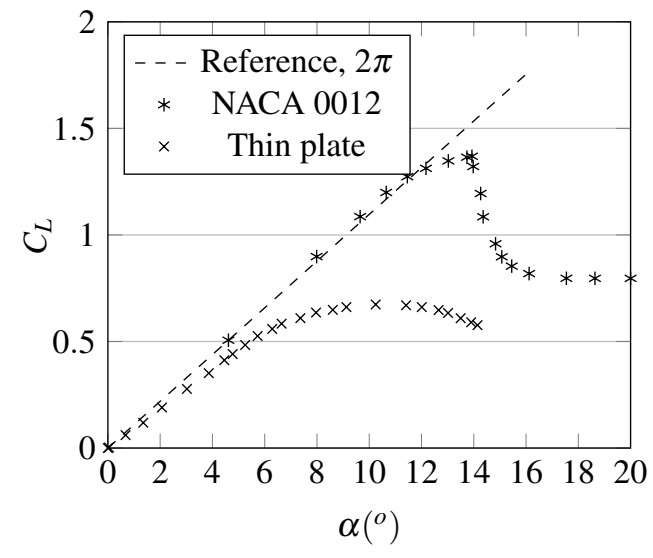

(a)

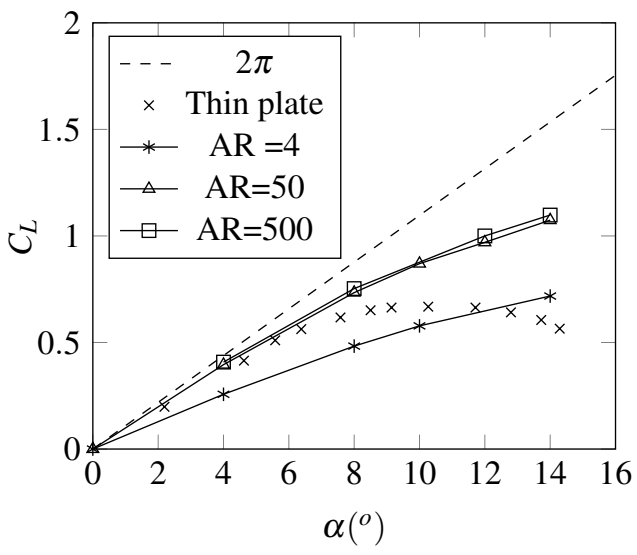

(b)

Figure 6. (a) Quasy-static $C_{L} \times \alpha$ curves. (b) Results obtained with present approach, without stall limitations.

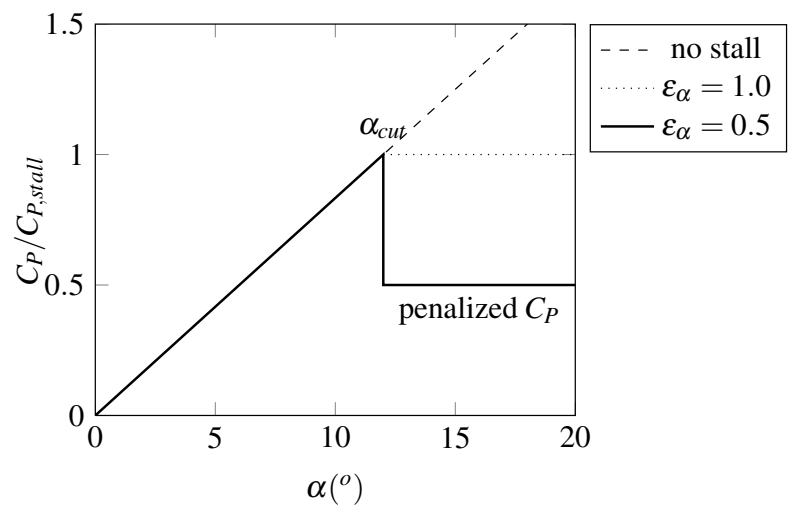

Figure 7. Schematic of penalized $C_{P}$ as function of panel effective angle-of-attack.

In summary, the presented strategy considers mainly the hysteretical behavior associated to potential flow effects. The separation effects is included as quasi-steady stall behavior based on the principle of the instantaneous lift efficiency loss, as far as the panel effective angle of attack $\alpha_{e}$ reaches $\alpha_{c u t}$, i.e., the airfoil static stall angle of attack.

\section{Numerical implementation}

The choice for the coupling method is the basis for the framework definition. Due to the characteristics of the aerodynamic solver, the time step tends to be larger than the required by the structural model, what creates difficulties for the use of implicit coupling methods. The choice comes then for an explicit method with subcycling in the structural part, contrary to what was used by reference ${ }^{14}$, in which subcycling in the aerodynamic part was used. In this way, inside the global time loop there is a structural-only time loop, in which the structural solver is called using a smaller time step. This has shown to be necessary, since the time step used for the aerodynamic model is fixed to comply with an uniform wake and wing discretization. In this case, the time is increased inside the structural loop with a time step $\Delta t_{s}$ until it reaches the next aeroelastic time step $t+\Delta t$. The value of $\Delta t_{s}$ is defined independently of the global $\Delta t$ and is based only in characteristics of the structural model.

The formulation is included into an aeroelastic simulation framework composed by a driver code, called IAE/ASAero, with the UVLM embedded in it, and coupled to UM/NLAMS, that is called as an external library. The UVLM has been implemented based on the codes available in ${ }^{24}$ but using $\mathrm{c}++$ implementation, in which the use of object oriented programming facilitates the data management. The model is constructed using a single text input file, similar to those used by commercial softwares like NASTRAN ${ }^{T M} 28$ or ZAERO ${ }^{T M}{ }^{29}$. In the present case, both the structural and the aerodynamic models are defined, as well as the coupling information and the simulation parameters. For the present work, some modifications have been added to the CSD solver, what became version 2.0 of UM/NLAMS. The most important is the capability of dealing with different number of materials, including laminated composites with an arbitrary number of different laminas. The formulation for that was already included in the paper of Khosravi et al. ${ }^{22}$ but had not been implemented computationally. Among others, version 2.0 has the following modifications compared with 
v1.1: (a) reading of a single input file containing all the information about the analysis; (b) compilation as a library (libnlams.a). This allows the program to be maintained as unique core, not being necessary to create new functions to comply with different codes. It also facilitates the evolution of the code as a single core since all the versions are unified, and there is no need to call each function separately, but only an interface function. Besides that, the code can be distributed as a "black-box", if necessary; (c) analysis of laminated composite plates without restriction in the number of layers; also: use of both isotropic and orthotropic material and use of various elements properties and materials in the same model.

A flowchart with the aeroelastic time response simulation is seen on Fig. 8. The definition of each analysis phase is established by means of an input file in ascii format. The vehicle state is then calculated at each time step inside the "Prescribed Motion" block. The UM/NLAMS is called from an interface function, that handles all the necessary information to be passed to the structural solver.

The complete aeroelastic framework is divided into two main blocks: the first is a pre-processing phase, when memory is allocated, and the initial conditions are established. For this initial phase, the time step is set to $k=-1$, which indicates to different modules that it is only a initialization step. The next block begins with the time step set to $k=0$, and the time is $t=k d t=0$. At each time step a prescribed motion module is called, defining the instantaneous system state variables, including the rotating frame transformation and skew matrices used by both structural and aerodynamic solvers. Once the internal structural time loop is completed, the displacement and velocities are transported to the aerodynamic model, and the aerodynamic solver is called. The pressures are transformed in nodal forces, and the next time step is ready to begin. Before that, all necessary information is recorded for post-processing. After completing the time loop it is only necessary to deallocate memory and write log files.

It has been identified the need for a restart procedure, in which a first simulation is performed to achieve a converged steady-state solution. From this converged solution, different types of perturbations can be easily applied and the nonlinear behavior of laminated composites plates properly investigated.
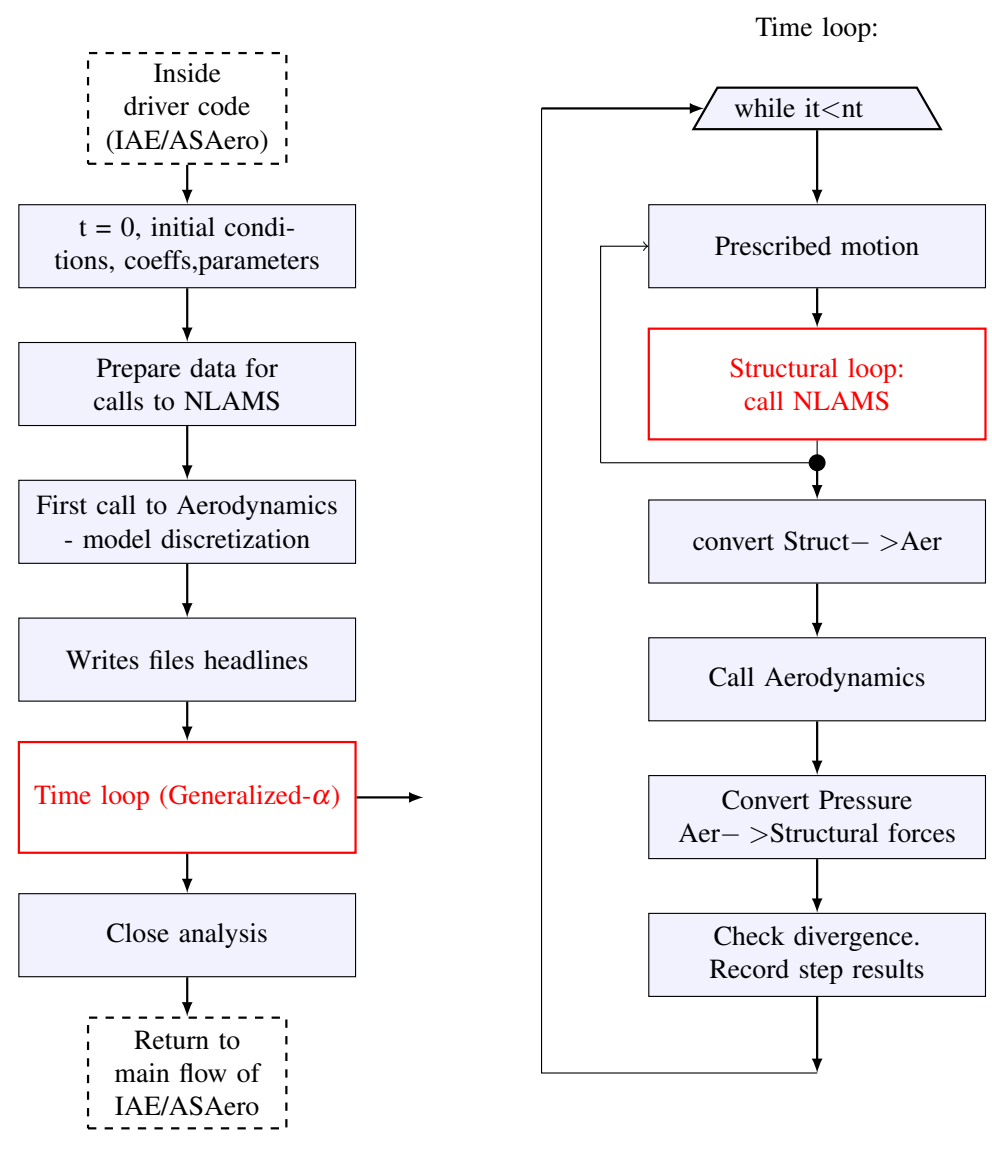

Figure 8. Aeroelastic simulation frame with coupled UM/NLAMS and IAE/ASAero.

\section{A. Aerodynamic model discretization}

Special attention is given to the aerodynamic model definition and construction. The information handling is important for an efficient computational code, and in the present case special attention has been given to that. The model is 
constructed using a single text input file, similar to those used by NASTRAN or ZAERO. In the present case, both the structural and the aerodynamic models are defined, as well as the coupling information and the simulation parameters. The aerodynamic model is defined using the concept of aerodynamic macro-elements (AME), which are continuous surfaces that will be divided into a finite number of aerodynamic panels. These macr elements are associated with a set of structural FE, and an internal routine identifies which are the nodes contained in both sides. The interpolations are then applied separately to each AME/FE set.

The loading results are dependent on the wake panel chord and the shedding procedure. It was noticed that the same wing panel chord should be used in the wake panel. Taking into consideration a wing that moves in the $\mathbf{e}_{I 1}$ direction at a constant velocity $\mathbf{v}$, the wing trailing edge suffers a displacement equal to $x_{1}=v_{r e f} \Delta t$ and the shed wake vortices have a chord value of $x_{1}$. To maintain the wake panel chord homogeneous in the complete wake, the time step has to be defined as

$$
\Delta t=\frac{1}{V_{r e f}}\left(\frac{c}{n_{b x}}\right)
$$

where $c$ is the wing chord and $n_{b x}$ is the number o bound panels in the chordwise direction. The total wake length, $L_{w}$, is established based on a desired number of main chords: $L_{w}=n_{c} c$, where $n_{c}$ is the number of chords.

(a)

Macro element

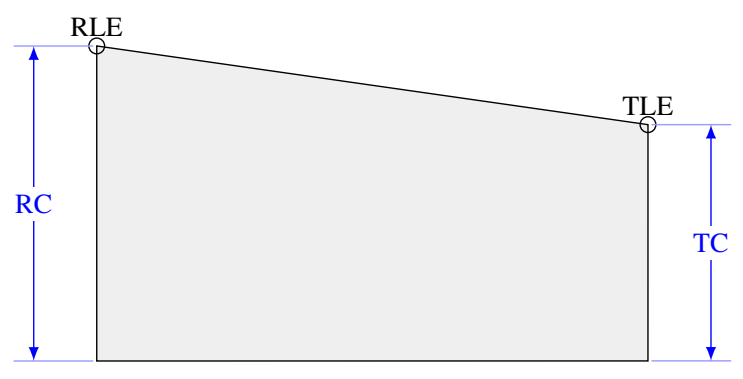

(b)

Lifting surface discretization: panels and GRIDA.

$i_{A}$ points in GRIDA

j panels

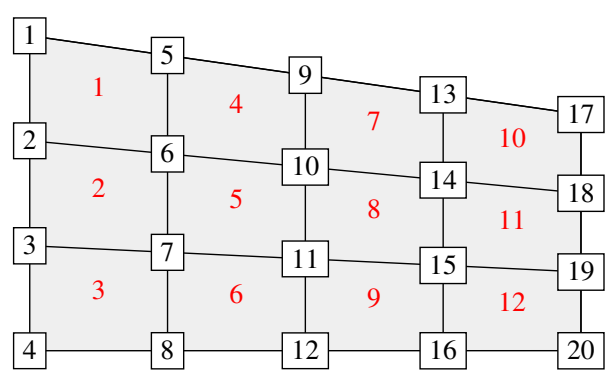

(c)

Bound vortex-ring elements definition: GRIDB.
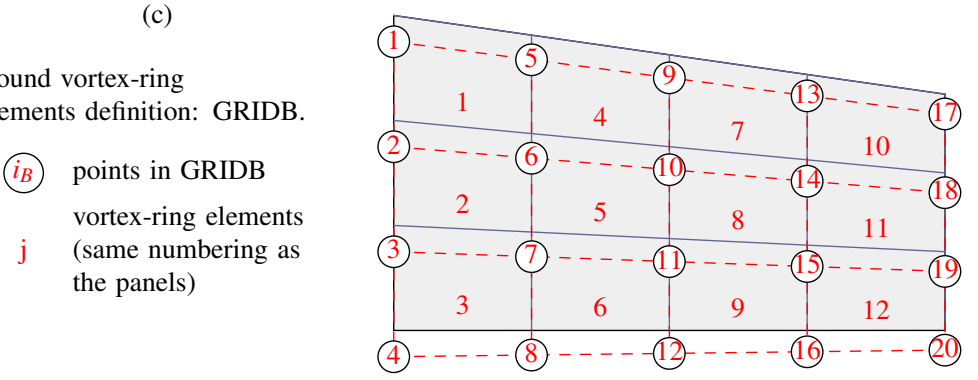

Figure 9. Discretization steps in the Vortex-Ring method, defined by coordinate of root leading egde (RLE), tip leading edge (TLE) points, root (RC) and tip (TC) chords.

The wake shedding follows a modified procedure, compared to the version described in ${ }^{24}$. At $t=0$, all the wake points are concentrated at the wing trailing edge. As the wing starts moving in the $-\mathbf{x}_{1}$ direction, the line of wake points attached to wing follows it, and the rest remain in the initial position. At $t=2 d t$ the second line of wake points follows the wing, too, and so forth. After a number of time steps equal to the number of wake points in the chordwise direction $(n p w x)$, all the wake is already following the wing.

A special procedure is used to evaluate $\Delta \Gamma_{i}$ and $\Delta \Gamma_{j}$ in Eq. (33), because they depend on the type of discretization used to model the aerodynamic panels. For example, in the case of multiple panels and panels placed in the starboard side, the numbering may be counting from a different position. The variations $\Delta \Gamma_{i}$ and $\Delta \Gamma_{j}$ are then established based on the panel position inside the macro element. 


\section{Numerical studies}

The numerical studies are divided into verification and investigation cases. First, results for the validation of aerodynamic and aeroelastic formulations are presented. Next, investigation of the nonlinear aeroelastic behavior of a composite flat plate wing model is developed.

\section{A. Verification of UVLM formulation}

For verification purposes, the example 1 of chapter 13 of $^{24}$ is used. This is an example of steady-state flight, with a solution for transient lift of a rigid wing. It means that no flight dynamics or aeroelasticity is taken into account, but the wing remains fixed and undeformed. The results are presented for a wing with the following parameters: rigid flat rectangular wing; aspect ratio from 4 to $200(=\infty)$; angle of attack of $5^{\circ} ; v_{r e f}=10 \mathrm{~m} / \mathrm{s}$ and chord of $1 \mathrm{~m}$; discretization 13 panels along the span and 4 along the chord.

In the first time step the gradient $\partial \Phi / \partial t$ is large, and convergence is achieved soon after that. The suggested value for the time step in ${ }^{24}$ is $\left(v_{r e f} \Delta t\right) / c=1 / 16$. However, the numerical investigation showed a dependency of the time step with the the size of the wake panel exists. It is necessary to adjust the time step value so that the chord of each wake panel has to be the same of the wing panels, using the formula given in Eq. (38). Results showed good agreement with the ones given in ${ }^{24}$, as seen in Figure 10.

The UVLM based aeroelastic framework is validated through comparison with experimental results published in ${ }^{9}$. Those experiments were made in a water tunnel, with Reynolds number $R e=30 \cdot 10^{3}$, flow velocity $v=0.3 \mathrm{~m} / \mathrm{s}$, and fluid density of $\rho_{\text {water }}=1000 \mathrm{~kg} / \mathrm{m}^{3}$. The reduced frequency, defined as $k_{G}=(\pi f c) / U_{\infty}$, varies from 0.25 to 2.00 .

A comparison of phase and amplitude lags are shown in Fig. 11, containing results for two different values of Elasticity modulus $E$ : $210 \mathrm{GPa}$ (called flexible) and $70 \mathrm{GPa}$ (called highly flexible). Results for $210 \mathrm{GPa}$ in general are good, specially for $k_{G}<1.5$. For the flexible model, tip vertical displacement results for $k_{G}=1.82$ are depicted on Fig. 12. At this frequency, both amplitude and phase lag are already deviated from reference values, but still show the general behavior trend. It can be concluded that higher reduced frequencies should be avoided when using the present formulation.

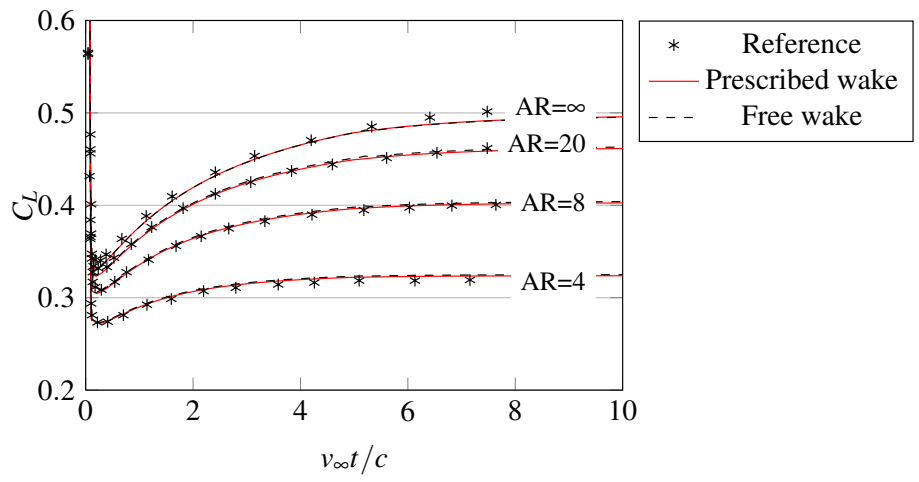

Figure 10. (a) Comparison of $d C_{L} / d \alpha$ for an increasing value of aspect ratio. (b) Convergence of $C_{L}$ with time step. Reference values from $^{24}$.
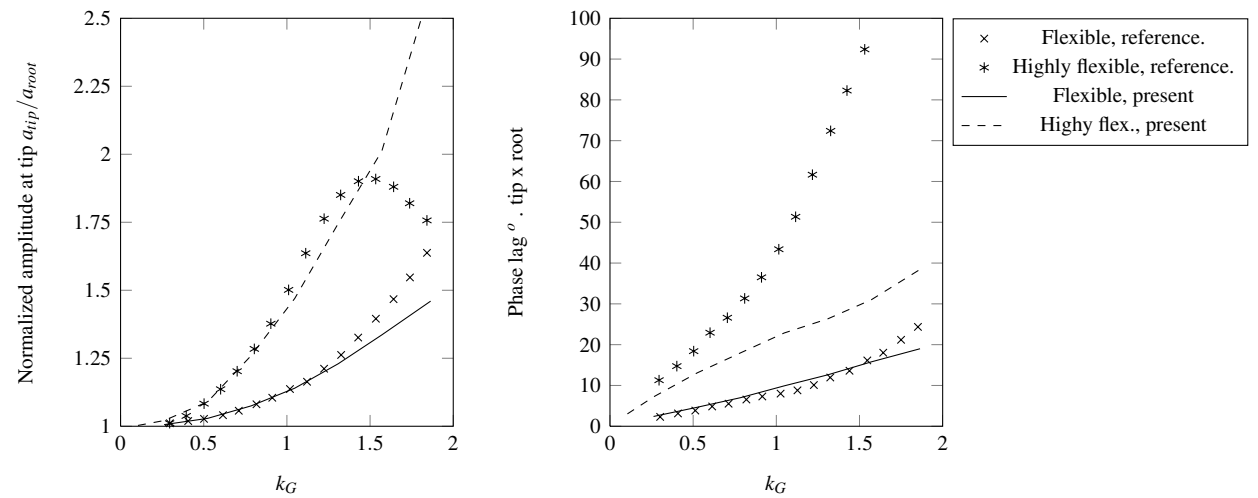

Figure 11. Phase and amplitude comparison. Reference values from ${ }^{9}$. 


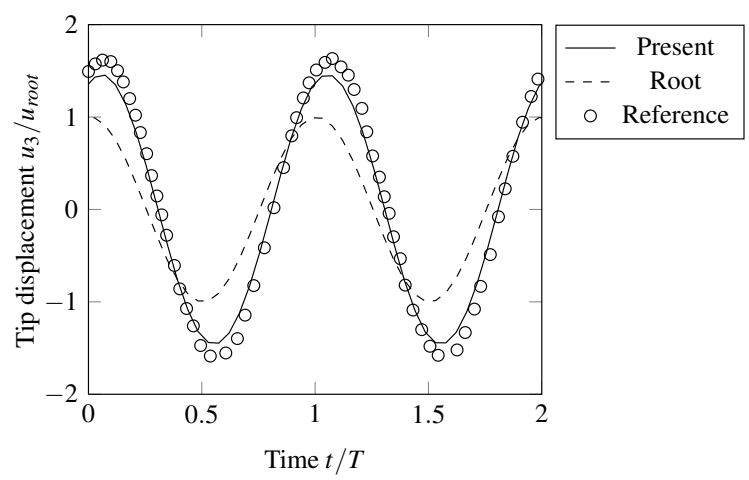

Figure 12. Displacement response of flexible model for $k_{G}=1.82$. Reference values from ${ }^{9}$.

\section{B. Study of proposed stall model}

To observe the stall effects, a hysteretic variation of the loading as a function of the quasi-steady angle of attack is used. The response of a rigid flat plate is obtained by applying a sinusoidal variation to the nominal angle-of-attack:

$$
\alpha=\alpha_{A} \sin \left(\omega_{A} t\right)
$$

where $\alpha_{A}$ is the oscillation amplitude, $\omega_{A}=2 \pi f_{A}$, the oscillation frequency in $\mathrm{rad} / \mathrm{s}$, with $f_{A}$ given in Hz. The results shown in the following were obtained for $\alpha_{A}=15^{\circ}$ and values of $f_{A}$ from 0.25 to $2.0 \mathrm{~Hz}, v_{\text {ref }}=10 \mathrm{~m} / \mathrm{s}$ and a chord of $1 \mathrm{~m}$. Also, in all simulations, $\alpha_{c u t}$ has been simply set to $12^{\circ}$ and $\varepsilon_{\alpha}$ to 0.5 .

Figure 13 shows hysteresis loops of a moderate aspect ratio flat plate $(\mathrm{AR}=10)$, comparing with a $C_{L} \times \alpha$ curve of a thin plate of infinite span. It is possible to observe that a hysteresis is established around the quasi-steady response. The post-stall behavior is very dependent on the chosen value of $\varepsilon_{\alpha}$. The selected approach allows further refinement based on test data, in the same sense done by the semi-empirical stall models available in the literature ${ }^{26}$. Next, the same flat plate with $\mathrm{AR}=10$ is subjected to different $f_{A}: 0.25,0.5$ and $1.0 \mathrm{~Hz}$, with results shown in Fig. 14 and 15 . It is possible to note that the hysteresis effects become more evident as the oscillation frequency increases. On the other hand, very small $f_{A}$ approximate the response to a quasi-steady behavior.

The influence of aspect ratio is related to the $C_{L}$ magnitude and hysteretic loop shape due to the wing tip vortex shedding. Figure 15 shows different hysteresis loops for $A R=4$ and $A R=10$. This behavior is justified by relevant three dimensional effects. As far as the aspect ratio decreases, the elliptic shape of the hysteretic loop tends to a circle. This is due to the an unsteady induced normalwash locally changing the effective angle of attack, in other words, anticipate or delay the panel local effective angle of attack.

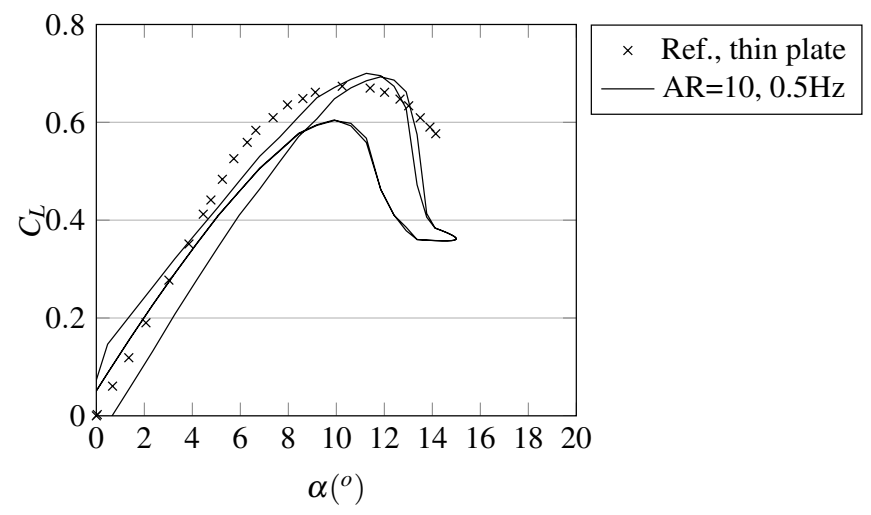

Figure 13. Comparison of a quasi-steady $C_{L} \times \alpha$ curve for a thin plate and a hysteresis loop for an oscillating flat plate $\left(\mathbf{A R}=\mathbf{1 0}, f_{A}=0.5 H z\right)$. 


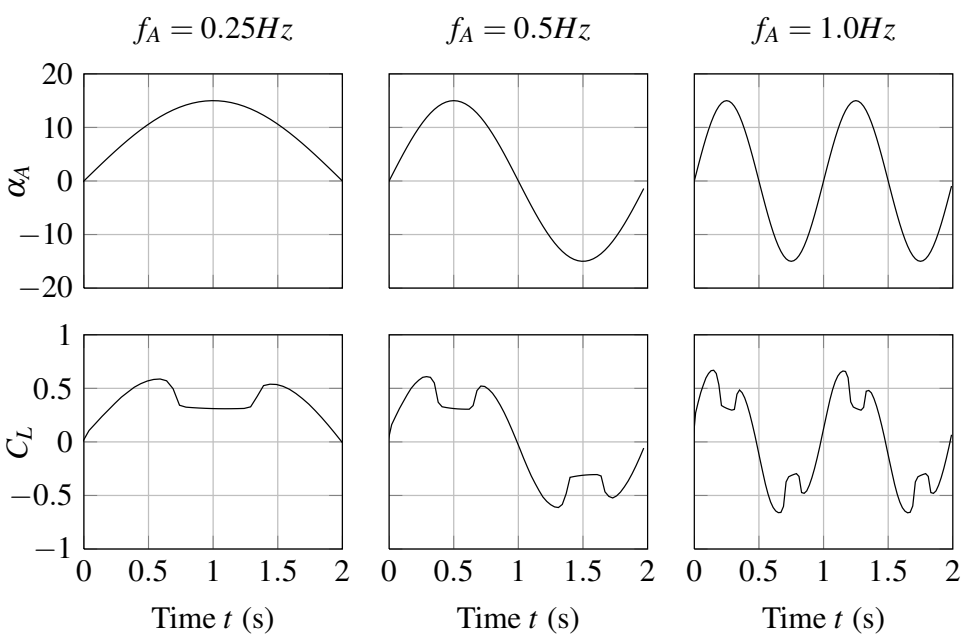

Figure 14. Lift coefficient results for a flat plate under a cyclic variation of prescribed angle of attack $\alpha$ including a stall model.
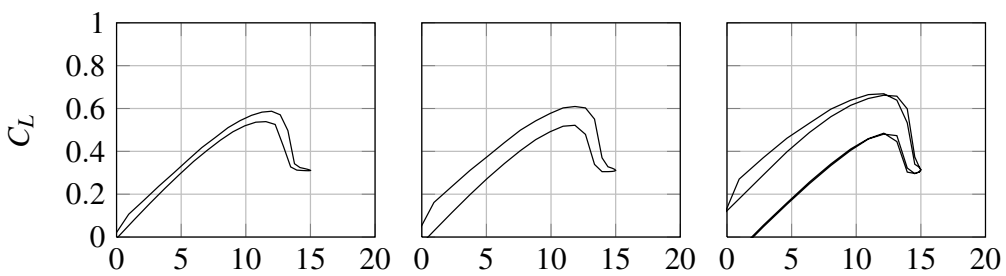

$\mathrm{AR}=10, f_{A}=0.25 \mathrm{~Hz}$
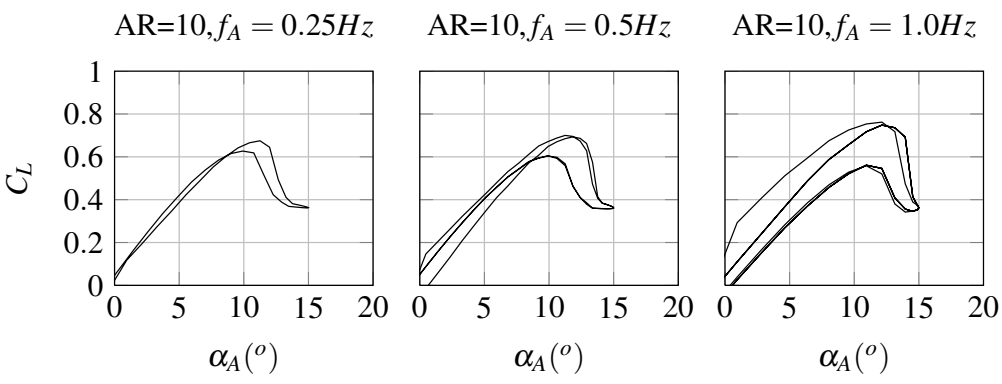

Figure 15. Hysteresis loops for oscillating flat plates under $\alpha=\alpha_{r} \sin \left(\omega_{r} t\right)$ and different parameters.

\section{Single wing composite plate model}

A simple flat plate model is used as to illustrate the ability of the present framework to capture LCO behavior associated to large displacements and composite material properties. The models here investigated are similar to those in ${ }^{16}$, but now using properties of carbon fiber. Only numerical results are presented as a guidance for future experimental setup design. The properties of the model under investigation are summarized in Tab. 1. In this case, the material orientation angle $\theta_{k}$ is an investigation parameter, since one goal is to identify the influence of material orientation in the LCO time response results. For simplicity and reduction of total model parameters, only one orientation value is used for the whole plate. Model dimensions are seen in Fig. 16(a) and an example of different orientations in the same coordinate system, in Fig. 16(b). 


\begin{tabular}{|llll|ll|}
\hline Model: & \multicolumn{3}{l|}{ Material properties: } & \multicolumn{2}{l|}{ Aerodynamic model: } \\
dimensions $(\mathrm{m}):$ & $0.3 \times 0.1$ & $E_{1}(\mathrm{GPa}):$ & 128.0 & $\rho\left(\mathrm{kg} / \mathrm{m}^{3}\right):$ & 1.225 \\
number of elements: & 150 & $E_{2}(\mathrm{GPa}):$ & 11.0 & $b_{\text {ref }}(\mathrm{m}):$ & 0.1 \\
number of nodes: & 96 & $G_{12}(\mathrm{GPa}):$ & 4.5 & \\
layup : & $2 \times\left(\theta_{k}\right)$ & $v:$ & 0.25 & \\
lamina thickness $(\mathrm{m}):$ & $5 \cdot 10^{-4}$ & $\operatorname{dens}\left(\mathrm{km} / \mathrm{m}^{3}\right):$ & 1545.0 & \\
total thickness $(\mathrm{m}):$ & $1 \cdot 10^{-3}$ & & & \\
\hline
\end{tabular}

Sudden acceleration simulations were performed with the goal of observing the response to different material orientation $\theta_{k}$, forward flight velocity $v_{\infty}$ and root angle-of-atack $\alpha_{\text {root }}$. Based on the above stability results, $v_{\infty}$ is defined between 10 and $25 \mathrm{~m} / \mathrm{s}$. The prescribed motion at the wing root is

$$
\mathbf{u}=\left[\begin{array}{lll}
v_{\infty} t & 0 & 0
\end{array}\right]^{T}, \quad \Psi=\left[\begin{array}{lll}
0 & \alpha_{\text {root }}^{o}\left(\pi / 180^{\circ}\right) & 0
\end{array}\right]^{T}
$$

where $v_{\infty}$ and $\alpha_{\text {root }}$ are simulation parameters to be modified for the investigations described below. The resulting impulse from the sudden acelleration is the only pertubation applied to the model, but shows to be enough for the ongoing studies. The sections that follow present results and discussion for these tests.

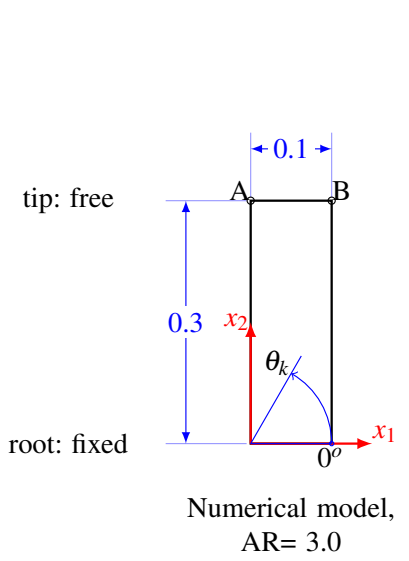

(a)
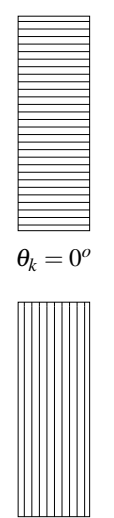

$\theta_{k}=90^{\circ}$

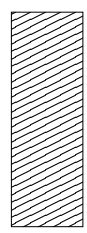

$30^{\circ}$

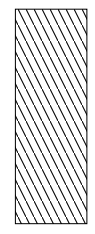

$120^{\circ}$

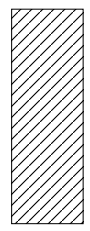

$45^{\circ}$

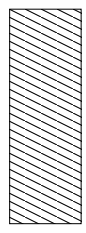

$165^{\circ}$

(b)

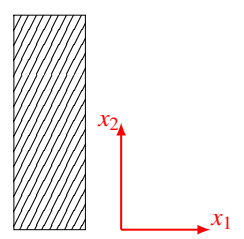

$60^{\circ}$

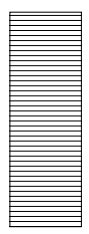

$180^{\circ}$

Figure 16. Flat plate model, made with carbon roving and epoxy. (a) Dimensions and position of analysis control points A and B. (b) Example of different orientations in the same coordinate system.

\section{Investigation of stall influence}

A first study consists in applying a stall model and compare different values of model parameter $\varepsilon$, given in Eq. (37). In a sudden acceleration simulation with $\alpha_{\text {root }}=10^{\circ}$, the tip displacement at point $\mathrm{A}$ is plotted in Fig. 17. The no stall condition leads to a highly damped response, with a steady condition rapdidly achieved. For the stall cases, LCO behavior is always observed, with increasing amplitudes for diminishing $\varepsilon$. The maximum amplitude is obtained for $\varepsilon=0.0$, since in this case the pressure on each bound vortex-ring element is set to zero. In all other cases, a certain pressure is mantained in the lifting surfaces. To define which value of $\varepsilon_{\alpha}$ should be used, hysteresis experiments are indicated to identify the post-stall behavior.

\section{Investigation of $\alpha_{\text {root }}$ and velocity increase influence}

The transient behavior of the flexible model subject to a sudden acceleration is studied. Based on the results from the linear aeroelastic stability analysis, velocities from 10 to $25 \mathrm{~m} / \mathrm{s}$ are considered in different simulations with $\alpha_{\text {root }}$ varying from $6^{\circ}$ to $14^{\circ}$. The same stall paremeters are used: $\alpha_{c u t}=12^{\circ}$ and $\varepsilon=0.5$. For this first investivation, only $\theta_{k}=0^{\circ}$ is used. The time step is adjusted to maintain the wake panel chord equal to the wing panels, following the procedure described in section IV. and Eq. (38). 

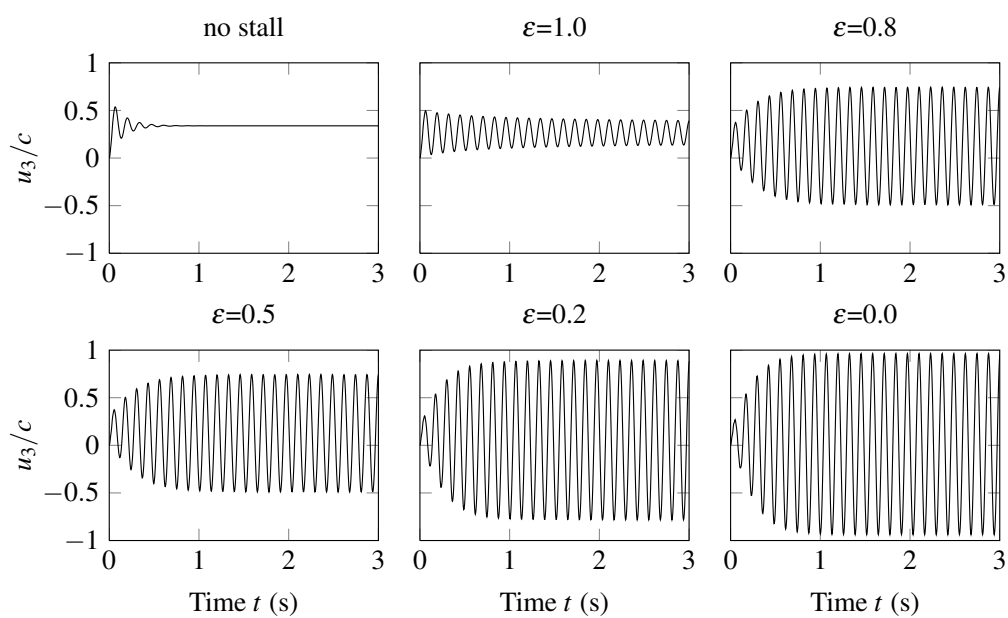

Figure 17. Time response of tip displacement (point A) for different values of stall parameter $\varepsilon$ in the stall model, with $\alpha_{\text {cut }}=12^{\circ}, \alpha_{\text {root }}=10^{\circ}$, $v_{\infty}=15 \mathrm{~m} / \mathrm{s}$.

Figure 18 shows the mean normalized vertical tip displacement for the present analysis. It is possible to observe that a converged stable condition is achieved for small $\alpha_{\text {root }}$ and velocities. For higher ones, LCOs are obtained, with larger amplitude for larger velocities. An interesting condition happens for $v_{\text {inf }}$ below $15 \mathrm{~m} / \mathrm{s}$ and $\alpha_{\text {root }}$ equal or above $\alpha_{\text {cut }}$ : a strong damping is observed. For a velocity of $25 \mathrm{~m} / \mathrm{s}, \mathrm{LCO}$ is already established for small $\alpha_{\text {root }}$, and even above $\alpha_{c u t}$ there is still a response with relatively large amplitude, what is caused by the high energy in the flow.

Figure 19 allows a closer look to the response for $v_{\infty}=15 \mathrm{~m} / \mathrm{s}$. The phase diagrams for the displacements $u_{1}$ and $u_{3}$ of the leading edge wing tip (point A) show that, for small angle $\alpha_{\text {root }}$, an equilibrium position is reached for both components. This does not happens for values above $08^{\circ}$, but is is seen that there is no large increase in the $u_{1}$ amplitude. The value of $u_{3}$, however, achieves large amplitudes, and nonlinear response components are observed when LCO is completely estalished, for $\alpha_{\text {root }}=8$ and $10^{\circ}$.

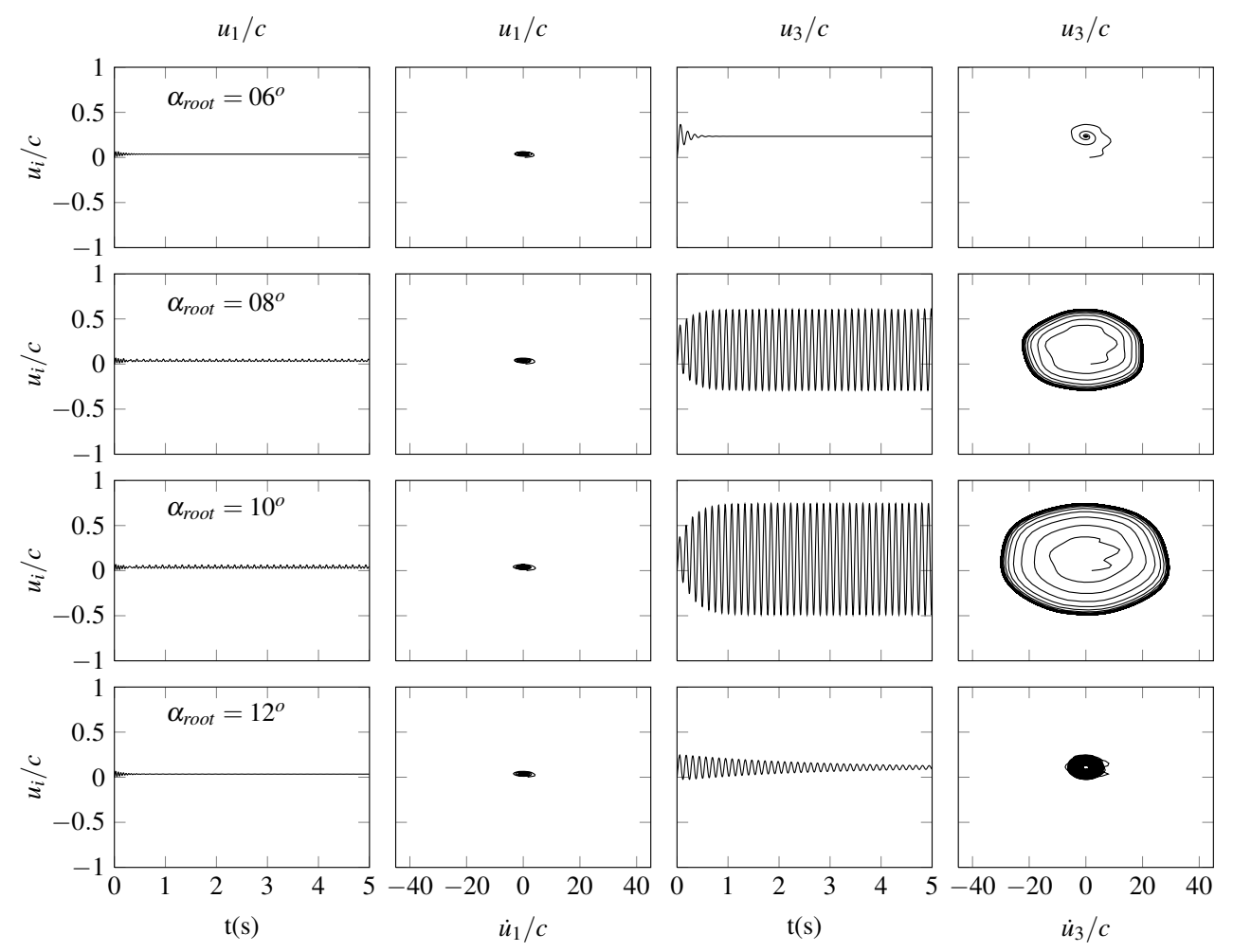

Figure 19. Time response and phase diagrams for displacement at point $\mathrm{A}$ for $\theta_{k}=0^{\circ}, v_{\infty}=15 \mathrm{~m} / \mathrm{s}, \alpha_{\text {root }}=06,08,10$ and $12^{\circ}$. 

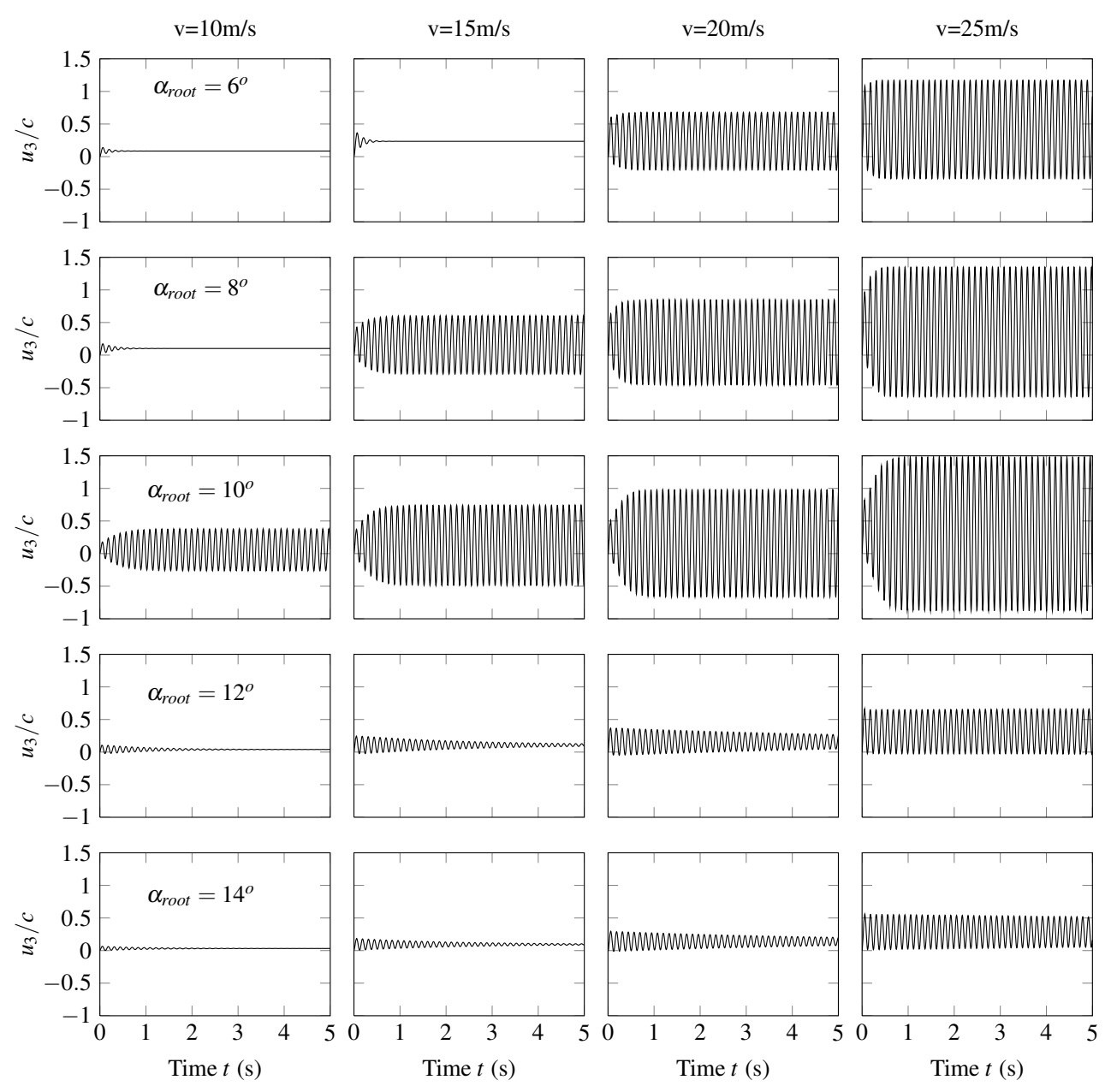

Figure 18. Time response of tip displacement (point A) for $v_{\infty}=10,15,20$ and $25 \mathrm{~m} / \mathrm{s}, \alpha_{\text {root }}=6,8,10,12$ and $14^{o}$. All models with $\theta_{k}=0^{o}$.

\section{Influence of Material Orientation}

Displacemente results for constant $v_{\infty}$ and different $\alpha_{\text {root }}$ and $\theta_{k}$ are shown in Fig. (20) for a 5 seconds interval. The investigation focus on the influence of $\theta_{k}$ on the response to the sudden acceleration case, in a simulation similar to the previous one. In this figure, each column corresponds to different material orientation, and each row correponds to different values of $\alpha_{\text {root }}$. The chosen velocity is $15 \mathrm{~m} / \mathrm{s}$, since it shows a larger amplitude variation and converged LCOs at higher $\alpha_{\text {root }}$. Values for $\theta_{k}$ from 0 to $180^{\circ}$ are investigated. A large influence of material orientation for the same $\alpha_{\text {root }}$ was verified.

A frequency domain methodology based on a Fast Fourier Transform algorithm combined with a Singular Value Decomposition approach for handling multiple output signals simultaneously is used to obtain the frequency spectrum for the different analyses. In Fig. (21), the displacement signal from points A and B (leading and trailing edge at tip) is used to show the effects on frequency and fundamental modes distribution. It is observed that the spectrum associated to LCO response (like $\theta_{k}=0^{\circ}, \alpha_{\text {root }}=8$ and $10^{\circ}$ ) show a main harmonic frequency followed by a set of super-harmonics.

Another way of observing the results is through plots of initial and final amplitudes as given in Fig. (22). These plots consist in a condensation of time results from the graphs in Fig. 20, where the initial amplitude is the value of $u_{3} / c$ obtained at the first peak and the final amplitude is the maximum value at the end of 5 seconds simulation.

In the case that the converged result is a steady state, as in $\alpha_{\text {root }}=6^{\circ}, \theta_{k}=0^{\circ}$ in Fig. 20, the final amplitude (ム) lies below the initial one ( $\square$ ). When a LCO is established, as for $\alpha_{\text {root }}=8^{\circ}, \theta_{k}=0^{\circ}$, the final amplitude now lies above the initial one. Despite lacking a transient information, the results show that a small variation in $\alpha_{\text {root }}$ is enough to take the wing to LCO response. This is mantained until the whole surface has the local $\alpha_{e}$ above $\alpha_{c u t}$, the limit angle in the stall model.

Regarding the material orientation, its influences shows in two main aspects: the beginning of the LCO behavior, and its amplitude. As expected, lamination angles near $90^{\circ}$ (oriented nearly in the spanwise direction - see Fig. (16)(b)) lead to smaller amplitudes and delayed LCO. On the other hand, an orientation aligned with the chordwise direction results in earlier LCO. The case of $\theta_{k}=165^{\circ}$ is special since it shows also a stronger influence on the fiber orientation and the free flow velocity. 

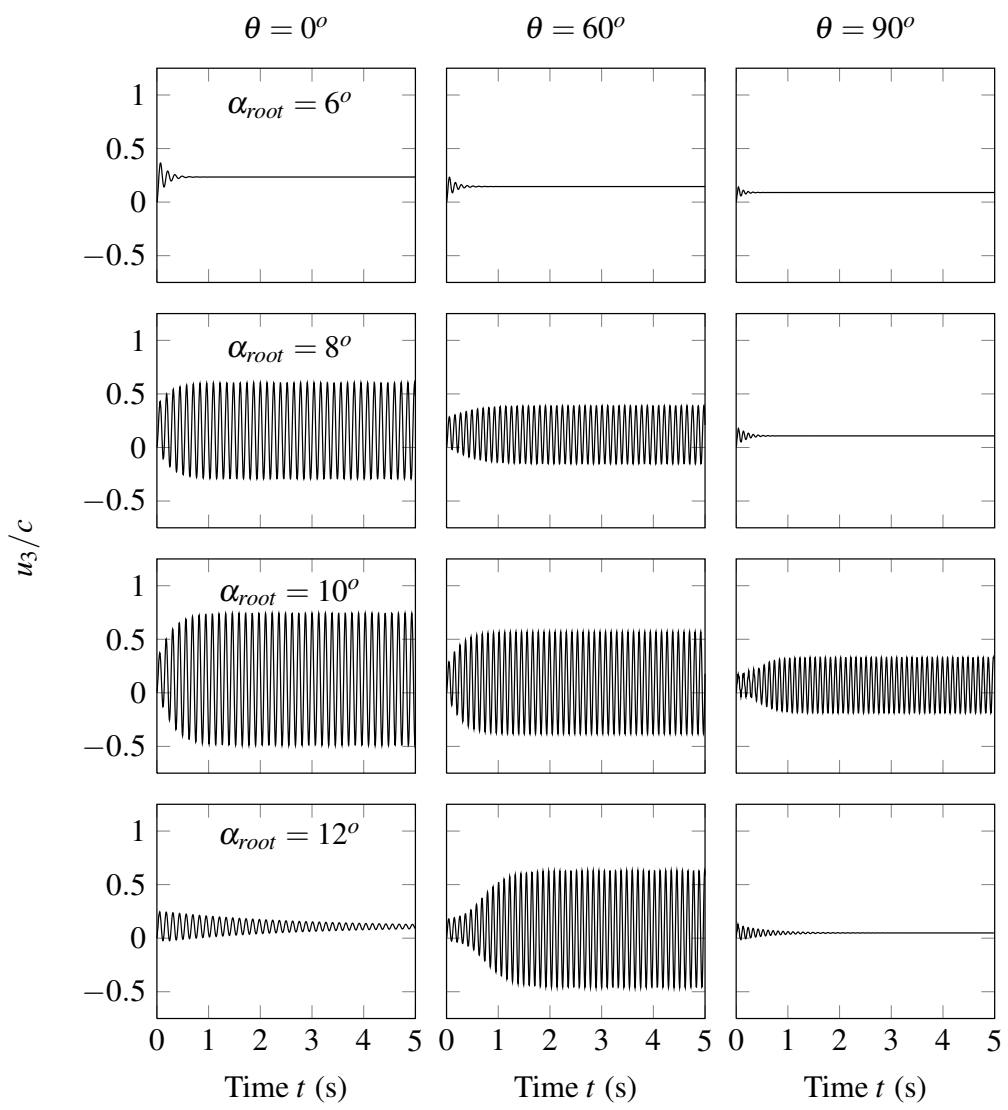

Figure 20. Time response for $v_{r e f}=15 \mathrm{~m} / \mathrm{s}$ and different $\theta_{k}$ and $\alpha_{\text {root }}$.
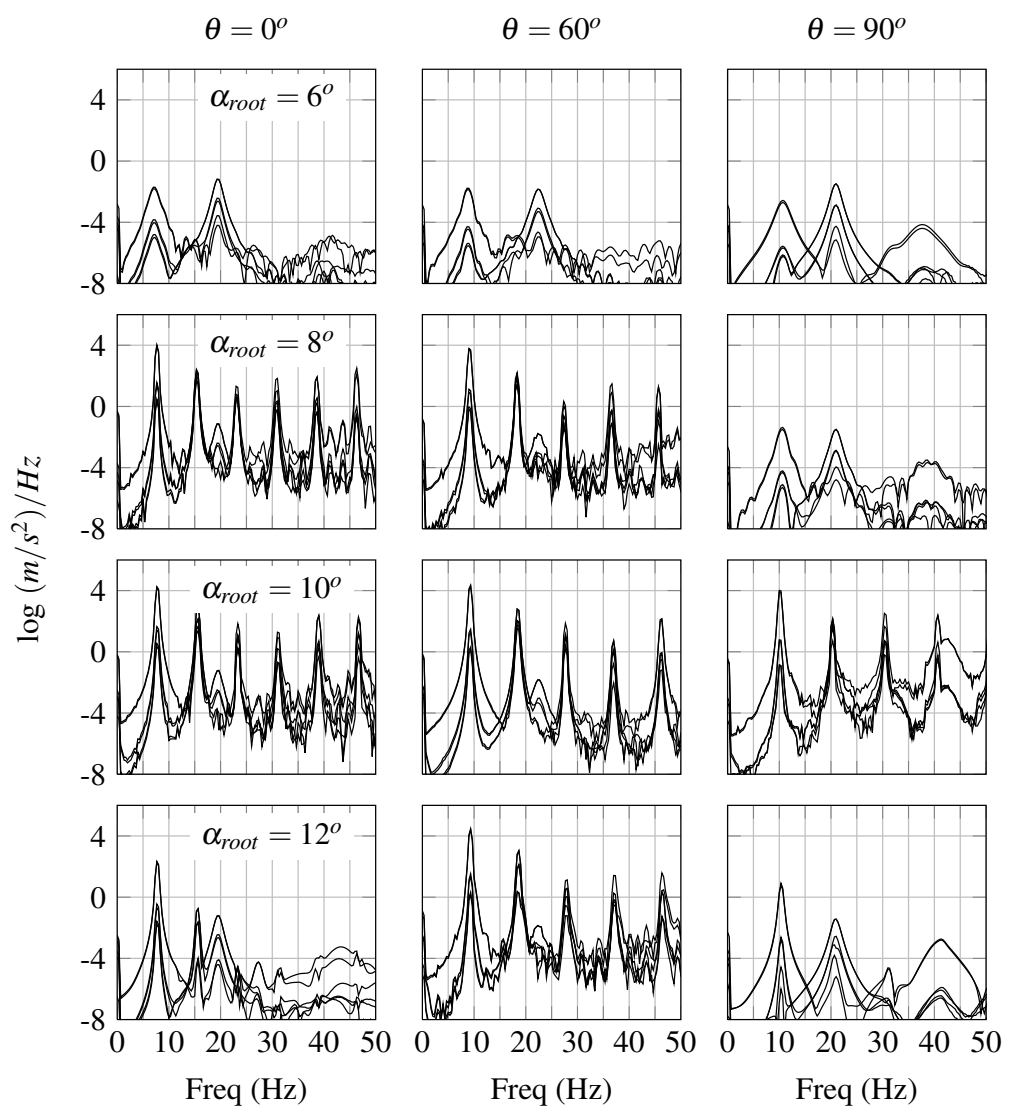

Figure 21. Frequency spectrum for $v_{r e f}=15 \mathrm{~m} / \mathrm{s}$ and different $\theta_{k}$ and $\alpha_{\text {root }}$. Amplitudes given a log of 10 

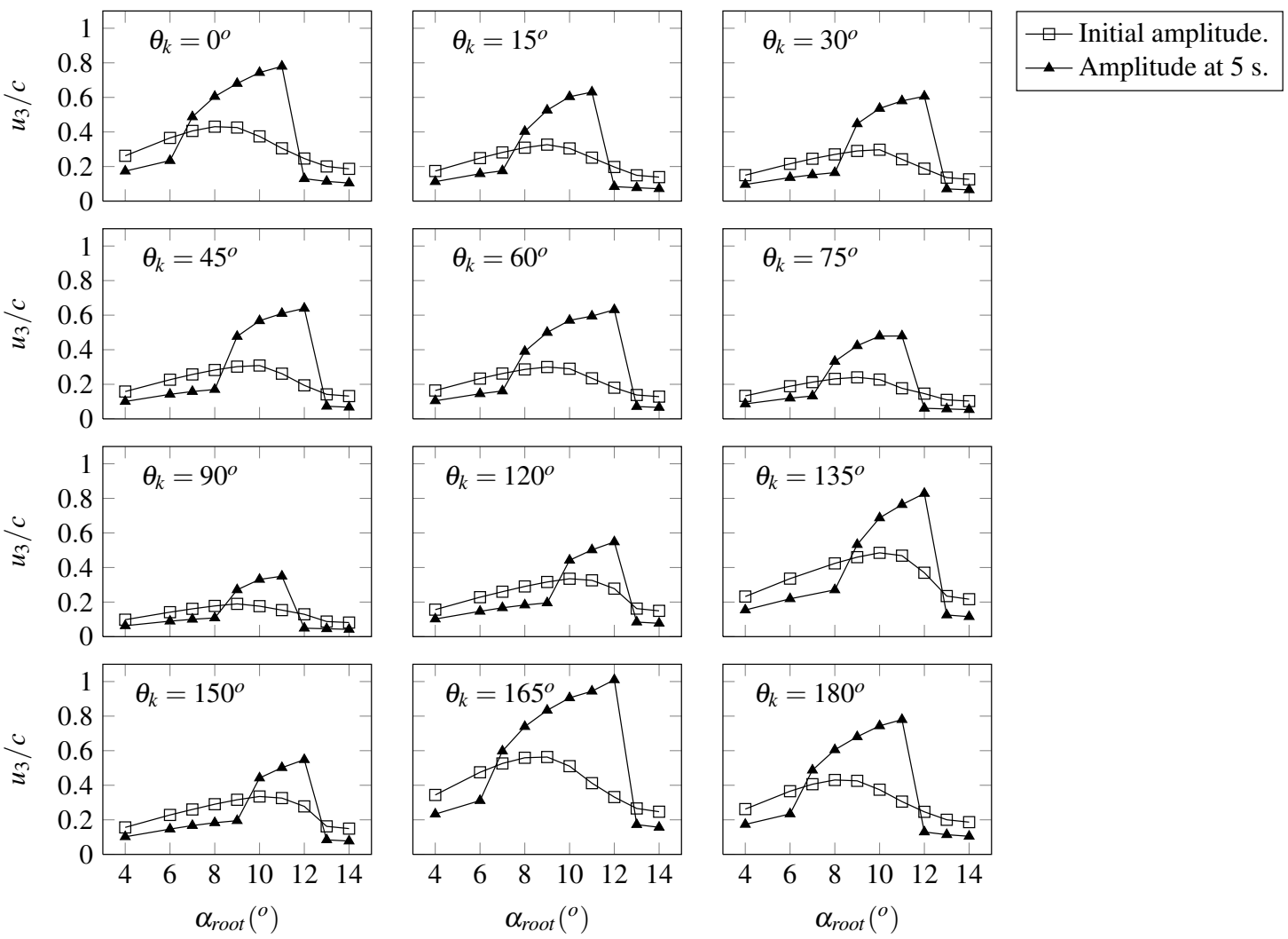

Figure 22. Initial and final amplitudes for a $5 \mathrm{~s}$ simulation $\left(v_{r e f}=15 \mathrm{~m} / \mathrm{s}\right.$ and different $\theta_{k}$ and $\alpha_{\text {root }}$ • )

\section{Conclusion}

An aeroelastic framework composed by a corotational shell finite element coupled with an unsteady vortex-lattice code was presented. This framework is intended for study of highly flexible plate-like wings subject to large displacements at a low computational cost. In the aerodynamic part of the problem, the addition of a stall model allowed the identification of LCO behavior, what is not possible when no stall is considered. Simulations of rigid flat plate showed that the lift coefficient can be well represented even around the stall region. Hysteresis loops simulations showed the ability to capture a lift lag expected for oscillating wings associated to separated flow effects. Comparisons with experimental results are necessary to define the two parameters defined for the stall model: the stall angle $\alpha_{c u t}$ and the post-stall penalty factor $\varepsilon_{\alpha}$. In the structural part, the inclusion of classical laminate theory is straightforward, since the corotational finite element formulation was already developed for that. The effects of material orientation in the time response is captured well in the flat plate simulation. LCO experiments for the different elastically tailored structures are underway.

\section{Acknowledgments}

The first and second authors acknowledge the partial support from the Instituto Nacional de Ciência e Tecnologia - Estruturas Inteligentes em Engenharia, INCT-EIE, the Conselho Nacional de Desenvolvimento Científico e Tecnológico, CNPq, and also the Fundação de Amparo à Pesquisa do Estado de Minas Gerais, FAPEMIG, from Brazil. The first author was also partially supported by the University of Michigan's Active Aeroelasticity and Structures Research Laboratory.

\section{References}

${ }^{1} \mathrm{Su}$, W. and Cesnik, C. E. S., "Nonlinear Aeroelastic Simulations of a Flapping Wing Micro Air Vehicle Using Two Unsteady Aerodynamic Formulations," 51 st AIAA/ASME/ASCE/AHS/ASC Structures, Structural Dynamics, and Materials Conference, No. AIAA-2010-2887, April 2010, pp. 1-22.

${ }^{2}$ Aono, H., Chimakurthi, S. K., Wu, P., Sällström, E., Stanford, B. K., Cesnik, C. E. S., Ifju, P., Ukeiley, L., and Shyy, W., "A Computational and Experimental Study of Flexible Flapping Wing Aerodynamics," 48th AIAA Aerospace Sciences Meeting Including the New Horizons Forum and Aerospace Exposition 4 - 7 January 2010, Orlando, Florida, 2010.

${ }^{3}$ Seber, G. and Bendiksen, O. O., "Nonlinear Flutter Calculations Using Finite Elements in a Direct Eulerian-Lagrangian Formulation," AIAA 
Journal, Vol. 46, No. 6, June 2008, pp. 1331-1341.

${ }^{4}$ Vest, M. S. and Katz, J., “Unsteady Aerodynamic Model of Flapping Wings," AIAA Journal, Vol. 34, No. 7, July 1996, pp. $1435-1440$.

${ }^{5}$ Fritz, T. E. and Long, L. N., "Object-Oriented Unsteady Vortex Lattice Method for Flapping Flight," Vol. 41, No. 6, 2004.

${ }^{6}$ Banerjee, S. P., Aeroelastic Analysis of Membrane Wings, Ph.D. thesis, Virginia Polythechnic Institute and State University, Blacksburg, August 2007.

${ }^{7}$ Stanford, B. and Beran, P., "An Updated Lagrangian Shell and Vortex Lattice Aeroelastic Framework for Flapping Wings," IFASD 2009, 2009 .

${ }^{8}$ Heathcote, S., Gursul, I., and Martin, D., "Flexible Flapping Airfoil Propulsion at Zero Freestream Velocity," AIAA Journal, Vol. 42, No. 11, Nov. 2004, pp. 2196-2204.

${ }^{9}$ Heathcote, S., Wang, Z., and Gursul, I., "Effect of spanwise flexibility on flapping wing propulsion," Journal of Fluids and Structures, Vol. 24, 2008, pp. 183-199.

${ }^{10}$ Wang, Z., Chen, P. C., Liu, D. D., and Mook, D. T., "Nonlinear- Aerodynamics/Nonlinear-Structure Interaction Methodology for a HighAltitude Long-Endurance Wing," Journal of Aircraft, Vol. 47, No. 2, 2010, pp. 556-566.

${ }^{11}$ Palacios, R., Murua, J., and Cook, R., "Structural and Aerodynamic Models in Nonlinear Flight Dynamics of Very Felixble Aircraft," AIAA Journal, Vol. 48, 2010, pp. 2648-2659.

${ }^{12}$ Wang, Z., Time-Domain Simulations of Aerodynamic Forces on Three-Dimensional Configurations, Unstable Aeroelastic Responses, and Control by Neural Network Systems, Ph.D. thesis, Virginia Polytechnic Institute and State University, 2004.

${ }^{13}$ Zhu, Q., "Numerical Simulation of a Flapping Foil with Chordwise or Spanwise Flexibility," AIAA Journal, Vol. 45, No. 10, 2007, pp. 24482457.

${ }^{14}$ Chimakurthi, S. K., Stanford, B. K., Cesnik, C. E. S., and Shyy, W., "Flapping Wing CFD/CSD Aeroelastic Formulation Based on a Co-rotational Shell Finite Element," 50th AIAA/ASME/ASCE/AHS/ASC Structures, Structural Dynamics, and Materials Conference 17th AIAA/ASME/AHS Adaptive Structures Conference 11th AIAA, 2009.

${ }^{15}$ Chimakurthi, S. K., Cesnik, C. E. S., and Stanford, B. K., "Flapping-Wing Structural Dynamics Formulation Based on a Corotational Shell Finite Element," AIAA Journal, Vol. 49, No. 1, January 2011, pp. 128-142.

${ }^{16}$ Aguiar, A. B., de Souza, C. E., and Marto, A. G., "Modelagem de uma Asa Aeroelástica Experimental em Compósito (in Portuguese)." VI Congresso Nacional de Eng. Mecânica, 18 a 21 de agosto de 2010 - Campina Grande - Paraíba - Brasil, 2010.

${ }^{17}$ Chung, J. and Hulbert, G. M., "A Time Integration Algorithm for Structural Dynamics With Improved Numerical Dissipation: The Generalized- $\alpha$ Method," Journal of Applied Mechanics, Vol. 60, No. 2, 1993, pp. 371.

${ }^{18}$ Crisfield, M. A., "A Consistent Co-Rotational Formulation for Non-Linear, Three-Dimensional, Beam-Elements," Comput. Methods Appl. Mech. Eng., Vol. 81, 1990, pp. 131-150.

${ }^{19}$ Shabana, A. A., Dynamics of Multibody Systems, Cambridge University Press, third edition ed., 2005.

${ }^{20}$ Duchon, J., Construtive Theory of Functions of Several Variables, chap. Splines Minimizing rotation-Invariant Semi-Norms in Sobolev Spaces, Springer, 1976, pp. 85-100.

${ }^{21}$ Cesnik, C. E. S., Smith, M. J., and Hodges, D. H., "Evaluation of Computational Algorithms Suitable for Fluid-Structure Interactions," Journal of Aircraft, Vol. 37, No. 2, 2000, pp. 282-294

${ }^{22}$ Khosravi, P., Ganesan, R., and Sedaghati, R., "Co-rotational Nonlinear Analysis of Thin Plates and Shells," International Journal of Numerical Methods in Engineering, Vol. 69, 2007, pp. 859-885.

${ }^{23}$ Reddy, J., Mechanics of Laminated Composite Plates: theory and analysis, CRC Press, Boca Raton, FL, 1997.

${ }^{24}$ Katz, J. and Plotkin, A., Low Speed Aerodynamics, Cambridge University Press, Cambridge, UK, 2nd ed., 2001.

${ }^{25}$ Katz, J., "Large-scale Vortex-Lattice Model for the Locally Separated Flow over Wings," AIAA 14th Fluid and Plasma Dynamics Conference, June 1981.

${ }^{26}$ Bierbooms, W., "A comparison between unsteady aerodynamic models," Journal of Wind Engineering and Industrial Aerodynamics, Vol. 39, No. 1-3, 1992, pp. 23-33.

${ }^{27}$ Theodorsen, T., "General theory of aerodynamic instability and the mechanism of flutter," Tech. Rep. NACA-TR-496, NACA, 1935.

${ }^{28}$ MSC, "MSC/NASTRAN: Handbook for Aeroelastic Analysis, v.1 (version 65)," Tech. rep., The MacNeal-Schwendler Corporation, 1987.

${ }^{29}$ ZONA Technology, editor, ZAERO Theoretical Manual, Version 8.0, ZONA Tech., 2007. 